\title{
Dynamics Analysis of a Stochastic Delay Gilpin-Ayala Model with Markovian Switching
}

\author{
Qiaoqin Gao, ${ }^{1}$ Zhijiang Luo, ${ }^{1}$ and Guirong Liu ${ }^{2}{ }^{2}$ \\ ${ }^{1}$ Department of Mathematics, Luliang University, Lishi, Shanxi 033001, China \\ ${ }^{2}$ School of Mathematical Sciences, Shanxi University, Taiyuan, Shanxi 030006, China \\ Correspondence should be addressed to Guirong Liu; lgr5791@sxu.edu.cn
}

Received 1 February 2019; Accepted 16 May 2019; Published 4 June 2019

Academic Editor: Giancarlo Consolo

Copyright (c) 2019 Qiaoqin Gao et al. This is an open access article distributed under the Creative Commons Attribution License, which permits unrestricted use, distribution, and reproduction in any medium, provided the original work is properly cited.

This paper considers a stochastic delay Gilpin-Ayala model with Markovian switching. Using Lyapunov method, we show existence and uniqueness of global positive solution. Then, by using Chebyshev's inequality, $M$-matrix method, and BDG's inequality, stochastic permanence and asymptotic estimations of solutions are studied. Finally, numerical simulations illustrate the theoretical results. Our results generalize and improve the existing results.

\section{Introduction}

Logistic equation is one of the most important and most widely used mathematical models to describe the growth of biological species. In [1], Gilpin and Ayala introduced the following generalized logistic equation (Gilpin-Ayala model):

$$
\frac{\mathrm{d} x(t)}{\mathrm{d} t}=x(t)\left(r-a x^{\lambda}(t)\right)
$$

where $x(t)$ stands for the population size; $r, a$, and $\lambda$ are positive constants. For more details, see $[1,2]$.

Population systems are often subject to environmental noise and there are different types of noise. Therefore, stochastic population systems have been studied extensively. See [3-9] and the references therein. In [8], by using Itô's formula and the exponential martingale inequality, the authors studied the stationary distribution, ergodicity, and extinction of the following stochastic Gilpin-Ayala model:

$$
\begin{aligned}
\mathrm{d} x(t)= & x(t)\left(r-a x^{\lambda}(t)\right) \mathrm{d} t+\sigma_{1} x(t) \mathrm{d} B_{1}(t) \\
& +\sigma_{2} x^{1+\lambda}(t) \mathrm{d} B_{2}(t) .
\end{aligned}
$$

Further, [9] obtained the stationary distribution and ergodicity of model (2) and improved the corresponding results in [8]. In addition, there is another type of environment noise, namely, colour noise, which can be demonstrated as a switching between two or more regimes of environment. See [10-18] and the references therein. Reference [11] considered the following stochastic Gilpin-Ayala model under regime switching:

$$
\begin{aligned}
\mathrm{d} x(t)= & x(t)\left[r(\gamma(t))-a(\gamma(t)) x^{\lambda}(t)\right] \mathrm{d} t \\
& +\sigma_{1}(\gamma(t)) x(t) \mathrm{d} B_{1}(t) \\
& +\sigma_{2}(\gamma(t)) x^{1+\theta}(t) \mathrm{d} B_{2}(t),
\end{aligned}
$$

where there exist $\lambda>0, \theta>0$, and studied persistence, extinction, nonpersistence, and global attractivity of (3). Further, [12] obtained the lower-growth rate and the uppergrowth rate of the positive solution of (3). Reference [13] considered the following stochastic Gilpin-Ayala population model with Markovian switching:

$$
\begin{aligned}
\mathrm{d} x(t)= & x(t)\left[r(\gamma(t))-a(\gamma(t)) x^{\lambda(\gamma(t))}(t)\right] \mathrm{d} t \\
& +\sigma_{1}(\gamma(t)) x(t) \mathrm{d} B(t),
\end{aligned}
$$

where the Gilpin-Ayala parameter $\lambda$ is also allowed to switch. In [13], the authors established the global stability of the trivial equilibrium state of (4). In addition, they obtained extinction, persistence, and existence of a stationary distribution of (4). 
On the other hand, many population systems depend on not only present states but also past states. See [19-26]. In [26], the authors considered a delay logistic model under regime switching

$$
\begin{aligned}
\mathrm{d} x & (t)=x(t) \\
\cdot & {[r(\gamma(t))-a(\gamma(t)) x(t)+b(\gamma(t)) x(t-\tau)] \mathrm{d} t } \\
& +\sigma_{1}(\gamma(t)) x(t) \mathrm{d} B(t) .
\end{aligned}
$$

They obtained stochastically ultimate boundedness, stochastic permanence, and extinction as well as asymptotic estimations of solutions.

Motivated by the works mentioned above, in this paper, we consider a more general delay stochastic Gilpin-Ayala model under Markovian switching

$$
\begin{aligned}
& \mathrm{d} x(t)=x(t) \\
& \cdot\left[r(\gamma(t))-a(\gamma(t)) x^{\lambda}(t)+b(\gamma(t)) x^{\lambda}(t-\tau)\right] \mathrm{d} t \\
& \quad+\sigma_{1}(\gamma(t)) x(t) \mathrm{d} B_{1}(t)+\sigma_{2}(\gamma(t)) x^{1+\theta}(t) \mathrm{d} B_{2}(t),
\end{aligned}
$$

with the initial conditions

$$
x(s)=\varphi(s)>0, \quad s \in[-\tau, 0] ; \gamma(0)=\iota,
$$

where $\tau>0, \varphi \in C\left([-\tau, 0], R_{+}\right) ; R_{+}=(0,+\infty) ; \lambda>0, \theta>$ $0 ; r, a: S \longrightarrow R_{+} ; b, \sigma_{1}, \sigma_{2}: S \longrightarrow R ; S=\{1,2, \ldots, m\}$.

Throughout this paper, let $\left(\Omega, F,\left\{F_{t}\right\}_{t \geq 0}, P\right)$ be a complete probability space with filtration $\left\{F_{t}\right\}_{t \geq 0}$, where $F_{t}$ is right continuous and $F_{0}$ contains all $P$-null sets. The one-dimensional Brownian motions $B_{1}(t)$ and $B_{2}(t)$ are defined in this space.

Let $\gamma(t)$ be a right-continuous Markov chain on the probability space, taking values in $S$, and with infinitesimal generator $Q=\left(q_{i j}\right) \in R^{m \times m}$ given by

$$
\begin{aligned}
& P\{\gamma(t+\delta)=j \mid \gamma(t)=i\} \\
& \quad= \begin{cases}q_{i j} \delta+o(\delta), & \text { if } i \neq j, \\
1+q_{i j} \delta+o(\delta), & \text { if } i=j,\end{cases}
\end{aligned}
$$

where $\delta>0, q_{i j} \geq 0$ is the transitions rate from $i$ to $j$ for $i \neq j$ and $q_{i i}=-\sum_{j \neq i} q_{i j}$ for each $i, j \in S$. We assume that the Markov chain $\gamma(t)$ is irreducible, and $B_{1}(t), B_{2}(t)$, and $\gamma(t)$ are independent under this condition; the Markov chain has a unique stationary (probability) distribution $\pi=$ $\left(\pi_{1}, \pi_{2}, \ldots, \pi_{m}\right) \in R^{1 \times m}$, which can be determined by solving the following equation:

$$
\pi Q=0,
$$

subject to $\sum_{i=1}^{m} \pi_{i}=1$ and $\pi_{i}>0, i \in S$.

For convenience, for any $f: S \longrightarrow R$, denote

$$
\begin{aligned}
& \widehat{f}=\max _{i \in S}\{f(i)\}, \\
& \check{f}=\min _{i \in S}\{f(i)\}, \\
& \bar{f}=\max _{i \in S}\{|f(i)|\} .
\end{aligned}
$$

For (6), we give the following conditions:
(A1) $\sigma_{2}(i) \neq 0$;

(A2) $\bar{b}-a(i)<\sigma_{2}(i)=0$;

(A3) $\bar{b}-a(i)=\sigma_{2}(i)=0, \lambda<1 / 2, r(i)<(1 / 4) \sigma_{1}^{2}(i)$;

(A4) $\bar{b}-a(i)=\sigma_{2}(i)=0, \lambda=1 / 2, \bar{b}+a(i)+r(i) \leq(1 / 4) \sigma_{1}^{2}(i)$;

(A5) $\bar{b}-a(i)<(1 / 4) \sigma_{2}^{2}(i)$;

(A6) $\bar{b}-a(i)=(1 / 4) \sigma_{2}^{2}(i), \lambda<1 / 2, r(i)<(1 / 4) \sigma_{1}^{2}(i)$;

(A7) $\bar{b}-a(i)=(1 / 4) \sigma_{2}^{2}(i), \lambda=1 / 2, \bar{b}+a(i)+(1 / 2) \sigma_{2}^{2}(i)+$ $r(i) \leq(1 / 4) \sigma_{1}^{2}(i) ;$

(A8) $\bar{b}<a(i)$;

(A9) $\bar{b}=a(i), \lambda<2 \theta+1 / 2, \sigma_{2}(i) \neq 0$;

(A10) $\bar{b}=a(i), \lambda<2 \theta+1 / 2, \sigma_{2}(i)=0, \lambda<1 / 2, r(i)<$ $(1 / 4) \sigma_{1}^{2}(i)$;

(A11) $\bar{b}=a(i), \lambda=2 \theta+1 / 2, \bar{b}+a(i)<(1 / 4) \sigma_{2}^{2}(i)$;

(A12) $\bar{b}=a(i), \lambda=2 \theta+1 / 2, \bar{b}+a(i)=(1 / 4) \sigma_{2}^{2}(i), 2 \theta<$ $1 / 2, r(i)<(1 / 4) \sigma_{1}^{2}(i)$;

(A13) $\bar{b}=a(i), \lambda=2 \theta+1 / 2, \bar{b}+a(i)=(1 / 4) \sigma_{2}^{2}(i), 2 \theta=$ $1 / 2, r(i)+(1 / 2) \sigma_{2}^{2}(i)<(1 / 4) \sigma_{1}^{2}(i)$.

(H1) $\lambda<2 \theta$; and for every $i \in S$, one of conditions (A1)(A4) holds;

(H2) $\lambda=2 \theta$; and for every $i \in S$, one of conditions (A5)(A7) holds;

(H3) $\lambda>2 \theta$; and for every $i \in S$, one of conditions (A8)(A13) holds;

(H4) $\lambda<2 \theta$; and for every $i \in S$, (A1) or (A4) holds;

(H5) $\lambda=2 \theta, \bar{b}<a(i)+(1 / 4) \sigma_{2}^{2}(i), i \in S$;

(H6) $\lambda>2 \theta, \bar{b}<\check{a}$;

(H7) there exists $u \in S$ such that for any $i \in S \backslash\{u\}, q_{i u}>0$;

(H8) $\sum_{i=1}^{m} \pi_{i} \beta(i)>0$, where $\beta(i)=r(i)-(1 / 2) \sigma_{1}^{2}(i), i \in S$;

(H9) $\bar{b}<\check{a}$.

\section{Uniqueness of Global Positive Solution}

As $x(t)$ in (6) denotes population size at time $t$, it should be nonnegative. Hence, we shall consider the existence and uniqueness of global positive solution of (6).

Theorem 1. If one of conditions (H1)-(H3) holds, then, for any initial value $\varphi \in C\left([-\tau, 0] ; R_{+}\right), \iota \in S$, there exists a unique solution $x(t)$ to (6) on $[-\tau,+\infty)$ and $x(t)$ will remain in $R_{+}$ with probability 1.

Proof. Since the coefficients of (6) are locally Lipschitz continuous, then there is a unique maximal local solution $x(t)$ on $\left[-\tau, \tau_{e}\right)$, where $\tau_{e}$ is the explosion time. Let $k_{0}>0$ satisfying

$$
\frac{1}{k_{0}}<\min _{-\tau \leq t \leq 0} \varphi(t) \leq \max _{-\tau \leq t \leq 0} \varphi(t)<k_{0} .
$$


For each integer $k \geq k_{0}$, define the stopping time

$$
\tau_{k}=\inf \left\{t \in\left[0, \tau_{e}\right): x(t) \notin\left(\frac{1}{k}, k\right)\right\} .
$$

Here, set inf $\emptyset=\infty$. Obviously, $\tau_{k}$ is increasing as $k \longrightarrow \infty$ and $\tau_{\infty}=\lim _{k \rightarrow+\infty} \tau_{k} \leq \tau_{e}$ a.s. If $\tau_{\infty}=\infty$ a.s., then $x(t) \in R_{+}$, for $t \geq 0$ and $\tau_{e}=\infty$ a.s. Thus, to prove this theorem, we need to prove $\tau_{\infty}=\infty$ a.s. Let

$$
V(x)=\sqrt{x}-1-\frac{1}{2} \ln x, \quad x>0 .
$$

Clearly, $V(x) \geq 0$ for any $x>0$. Applying Itô's formula to $V(x)$, we have

$$
\begin{aligned}
& \mathrm{d} V(x(t))=L V(x(t), x(t-\tau), \gamma(t)) \mathrm{d} t \\
& +\frac{\sqrt{x(t)}-1}{2}\left[\sigma_{1}(\gamma(t)) \mathrm{d} B_{1}(t)\right. \\
& \left.+\sigma_{2}(\gamma(t)) x^{\theta}(t) \mathrm{d} B_{2}(t)\right],
\end{aligned}
$$

where $L V: R_{+} \times R_{+} \times S \longrightarrow R$ is defined by

$$
\begin{aligned}
L V(x, y, i)= & -\frac{1}{4}\left(\frac{1}{2} x^{1 / 2}-1\right)\left[\sigma_{1}^{2}(i)+\sigma_{2}^{2}(i) x^{2 \theta}\right] \\
& +\frac{1}{2}\left(x^{1 / 2}-1\right)\left[r(i)-a(i) x^{\lambda}\right] \\
& +\frac{1}{2} b(i)\left(x^{1 / 2}-1\right) y^{\lambda}
\end{aligned}
$$

By Young's inequality,

$$
\begin{aligned}
& L V(x, y, i) \\
& \leq- \frac{1}{4}\left(\frac{1}{2} x^{1 / 2}-1\right)\left[\sigma_{1}^{2}(i)+\sigma_{2}^{2}(i) x^{2 \theta}\right] \\
&+\frac{1}{2}\left(x^{1 / 2}-1\right)\left[r(i)-a(i) x^{\lambda}\right]+\frac{1}{2} \bar{b} x^{1 / 2} y^{\lambda} \\
&+\frac{1}{2} \bar{b} y^{\lambda} \\
& \leq-\frac{1}{4}\left(\frac{1}{2} x^{1 / 2}-1\right)\left[\sigma_{1}^{2}(i)+\sigma_{2}^{2}(i) x^{2 \theta}\right] \\
&+\frac{1}{2}\left(x^{1 / 2}-1\right)\left[r(i)-a(i) x^{\lambda}\right]
\end{aligned}
$$

$$
\begin{aligned}
+ & \frac{1}{2} \bar{b}\left(\frac{1 / 2}{1 / 2+\lambda} x^{1 / 2+\lambda}+\frac{\lambda}{1 / 2+\lambda} y^{1 / 2+\lambda}\right) \\
+ & \frac{1}{2} \bar{b} y^{\lambda} \\
= & -\frac{1}{4}\left(\frac{1}{2} x^{1 / 2}-1\right)\left[\sigma_{1}^{2}(i)+\sigma_{2}^{2}(i) x^{2 \theta}\right] \\
+ & \frac{1}{2}\left(x^{1 / 2}-1\right)\left[r(i)-a(i) x^{\lambda}\right]+\frac{(1 / 2) \bar{b}}{1+2 \lambda} x^{1 / 2+\lambda} \\
+ & \frac{\bar{b} \lambda}{1+2 \lambda} x^{1 / 2+\lambda}+\frac{1}{2} \bar{b} x^{\lambda} \\
+ & \frac{\bar{b} \lambda}{1+2 \lambda}\left(y^{1 / 2+\lambda}-x^{1 / 2+\lambda}\right)+\frac{1}{2} \bar{b}\left(y^{\lambda}-x^{\lambda}\right) \\
\leq & F(x, i)+\frac{\lambda \bar{b}}{1+2 \lambda}\left(y^{1 / 2+\lambda}-x^{1 / 2+\lambda}\right) \\
& +\frac{\bar{b}}{2}\left(y^{\lambda}-x^{\lambda}\right),
\end{aligned}
$$

where

$$
\begin{aligned}
F(x, i)= & -\frac{1}{4}\left(\frac{1}{2} x^{1 / 2}-1\right)\left[\sigma_{1}^{2}(i)+\sigma_{2}^{2}(i) x^{2 \theta}\right] \\
& +\frac{1}{2} r(i)\left(x^{1 / 2}-1\right)+\frac{1}{2}(\bar{b}+a(i)) x^{\lambda} \\
& +\frac{1}{2}(\bar{b}-a(i)) x^{1 / 2+\lambda} .
\end{aligned}
$$

If one of conditions (H1)-(H3) holds, then $F(x, i)$ is bounded above; i.e., there exists $M_{1}>0$ such that $F(x, i) \leq M_{1}, x \in$ $R_{+}, i \in S$. Substituting these into (14) yields

$$
\begin{aligned}
& \mathrm{d} V(x(t)) \leq\left[M_{1}\right. \\
& +\frac{\lambda \bar{b}}{1+2 \lambda}\left(x^{1 / 2+\lambda}(t-\tau)-x^{1 / 2+\lambda}(t)\right) \\
& \left.+\frac{\bar{b}}{2}\left(x^{\lambda}(t-\tau)-x^{\lambda}(t)\right)\right] \mathrm{d} t+\frac{1}{2}\left(x^{1 / 2}(t)-1\right) \\
& \quad \cdot\left[\sigma_{1}(\gamma(t)) \mathrm{d} B_{1}(t)+\sigma_{2}(\gamma(t)) x^{\theta}(t) \mathrm{d} B_{2}(t)\right] .
\end{aligned}
$$

For any $T>0$ and $t \in[0, T]$, integrating both sides of (18) from 0 to $\tau_{k} \wedge t$ and taking the expectations yields

$$
\begin{aligned}
& E V\left(x\left(\tau_{k} \wedge t\right)\right) \leq V(\varphi(0))+E \int_{0}^{\tau_{k} \wedge t}\left[M_{1}\right. \\
& +\frac{\lambda \bar{b}}{1+2 \lambda}\left(x^{1 / 2+\lambda}(s-\tau)-x^{1 / 2+\lambda}(s)\right) \\
& \left.+\frac{\bar{b}}{2}\left(x^{\lambda}(s-\tau)-x^{\lambda}(s)\right)\right] \mathrm{d} s .
\end{aligned}
$$


In addition,

$$
\begin{aligned}
& E \int_{0}^{\tau_{k} \wedge t} x^{1 / 2+\lambda}(s-\tau) \mathrm{d} s \\
& \quad \leq \int_{-\tau}^{0} \varphi^{1 / 2+\lambda}(s) \mathrm{d} s+E \int_{0}^{\tau_{k} \wedge t} x^{1 / 2+\lambda}(s) \mathrm{d} s,
\end{aligned}
$$

and

$$
\begin{aligned}
E \int_{0}^{\tau_{k} \wedge t} x^{\lambda}(s-\tau) \mathrm{d} s \leq & \int_{-\tau}^{0} \varphi^{\lambda}(s) \mathrm{d} s \\
& +E \int_{0}^{\tau_{k} \wedge t} x^{\lambda}(s) \mathrm{d} s .
\end{aligned}
$$

Substituting (20) and (21) into (19) gives

$$
\begin{aligned}
E V\left(x\left(\tau_{k} \wedge t\right)\right) \leq & V(\varphi(0))+M_{1} T \\
& +\frac{\lambda \bar{b}}{1+2 \lambda} \int_{-\tau}^{0} \varphi^{1 / 2+\lambda}(s) \mathrm{d} s
\end{aligned}
$$

$$
+\frac{\bar{b}}{2} \int_{-\tau}^{0} \varphi^{\lambda}(s) \mathrm{d} s, \quad t \in[0, T] .
$$

Note that $x\left(\tau_{k}\right)=k$ or $x\left(\tau_{k}\right)=1 / k$. From (22), for any $T>0$,

$$
V(\varphi(0))+M_{1} T+\frac{\lambda \bar{b}}{1+2 \lambda} \int_{-\tau}^{0} \varphi^{1 / 2+\lambda}(s) \mathrm{d} s
$$

$$
\begin{aligned}
& +\frac{\bar{b}}{2} \int_{-\tau}^{0} \varphi^{\lambda}(s) \mathrm{d} s \geq E V\left(x\left(\tau_{k} \wedge T\right)\right) \\
& \geq E\left(I_{\left(\tau_{k} \leq T\right)} V\left(x\left(\tau_{k} \wedge T\right)\right)\right) \\
& =E\left(I_{\left(\tau_{k} \leq T\right)} V\left(x\left(\tau_{k}\right)\right)\right) \\
& \geq E\left[I_{\left(\tau_{k} \leq T\right)}\left(V(k) \wedge V\left(\frac{1}{k}\right)\right)\right]
\end{aligned}
$$

$$
\begin{aligned}
& =\left(V(k) \wedge V\left(\frac{1}{k}\right)\right) E\left(I_{\left(\tau_{k} \leq T\right)}\right) \\
& =\left(V(k) \wedge V\left(\frac{1}{k}\right)\right) P\left(\tau_{k} \leq T\right),
\end{aligned}
$$

which implies

$$
\limsup _{k \rightarrow \infty} P\left(\tau_{k} \leq T\right) \leq \lim _{k \rightarrow \infty} \frac{V(\varphi(0))+M_{1} T+(\lambda \bar{b} /(1+2 \lambda)) \int_{-\tau}^{0} \varphi^{1 / 2+\lambda}(s) \mathrm{d} s+(\bar{b} / 2) \int_{-\tau}^{0} \varphi^{\lambda}(s) \mathrm{d} s}{V(k) \wedge V(1 / k)}=0 .
$$

Then, $\lim _{k \rightarrow \infty} P\left(\tau_{k} \leq T\right)=0$. Further, $P\left(\tau_{\infty} \leq T\right)=0$. Since $T>0$ is arbitrary, we must have $P\left(\tau_{\infty}<\infty\right)=0$. That is, $\tau_{\infty}=\infty$ a.s. Therefore, Theorem 1 holds.

Corollary 2. Assume that (H9) holds. Then, for any initial value $\varphi \in C\left([-\tau, 0] ; R_{+}\right), \iota \in S$, there exists a unique solution $x(t)$ to (6) on $[-\tau,+\infty)$ and $x(t)$ will remain in $R_{+}$with probability 1.

Remark 3. If $\gamma(t) \equiv \varsigma \in S, t \in[0,+\infty) ; r(\varsigma)=r, a(\varsigma)=$ $a, b(\varsigma)=0, \sigma_{1}(\varsigma)=\sigma_{1}, \sigma_{2}(\varsigma)=\sigma_{2}, \theta=\lambda$, then (6) transforms to (2). Clearly, if $b(\varsigma)=0, \theta=\lambda$, then (H1) holds. Hence, Theorem 1 generalizes Lemma 2 in [9].

Remark 4. If $b(i)=0, i \in S$, then (6) reduces to (3). Clearly, if $b(i)=0, i \in S$, then one of conditions (H1)-(H3) holds. Hence, Theorem 1 generalizes Theorem 1 in [11].

\section{Stochastic Permanence}

Definition 5. Equation (6) is stochastically permanent if for any $\varepsilon \in(0,1)$, there exist constants $H=H(\varepsilon)>0$ and $\delta=$ $\delta(\varepsilon)>0$ such that for any solution $x(t)$ of (6),

$$
\begin{aligned}
& \liminf _{t \longrightarrow+\infty} P\{x(t) \leq H\} \geq 1-\varepsilon, \\
& \liminf _{t \longrightarrow+\infty} P\{x(t) \geq \delta\} \geq 1-\varepsilon .
\end{aligned}
$$

Lemma 6 (Lemma 3, [12]). If (H7) and (H8) hold, then there exists a constant $\alpha>0$ such that the matrix

$$
A(\alpha)=\operatorname{diag}\left(\xi_{1}(\alpha), \xi_{2}(\alpha), \ldots, \xi_{m}(\alpha)\right)-Q
$$

is a nonsingular $M$-matrix, where $\xi_{i}(\alpha)=(1+\theta) \alpha \beta(i)-$ $(1 / 2) \alpha^{2}(1+\theta)^{2} \sigma_{1}^{2}(i), i \in S$.

Lemma 7 (Theorem 2.10, [27]). If $A=\left(a_{i j}\right)_{n \times n} \in Z^{n \times n}$, then the following results are equivalent.

(1) A is nonsingular M-matrix.

(2) A is semipositive; that is, there is $x \gg 0$ in $R^{n}$ satisfying $A x \gg 0$.

Lemma 8. Assume that one of the following conditions holds:

(i) $0<p<1$ and (H4) holds

(ii) $p>0, \bar{b}<a(i)+(1 / 2)(1-p) \sigma_{2}^{2}(i), i \in S$ and (H5) holds

(iii) $p>0$ and (H6) holds

For any initial value $\varphi \in C\left([-\tau, 0] ; R_{+}\right), \iota \in S$, let $x(t)$ be the solution of (6) with the initial conditions (7). Then there exists $K_{1}(p)>0$ such that

$$
\limsup _{t \rightarrow+\infty} E\left(x^{p}(t)\right) \leq K_{1}(p) .
$$


Proof. From Theorem 1, if one of conditions (H4)-(H6) holds, then (6) exists as a unique global positive solution $x(t)$. Now, we prove Lemma 3 if (ii) holds. For $i \in S$, let

$$
\mu(i)= \begin{cases}\frac{1}{\tau} \ln \frac{(\lambda+p) a(i)-p \bar{b}+(1 / 2)(\lambda+p)(1-p) \sigma_{2}^{2}(i)}{\lambda \bar{b}}, & \bar{b}>0 \\ 1, & \bar{b}=0\end{cases}
$$

It follows from (ii) that $\mu(i)>0, i \in S$. Let $\beta>0$ satisfying $\beta<\min _{i \in S}\{\mu(i)\}$. Applying Itô's formula to $U=e^{\beta t} x^{p}$ yields

$$
\begin{aligned}
\mathrm{d} U(x(t), t)= & L U(x(t), x(t-\tau), t, \gamma(t)) \mathrm{d} t \\
& +p \sigma_{1}(\gamma(t)) e^{\beta t} x^{p}(t) \mathrm{d} B_{1}(t) \\
& +p \sigma_{2}(\gamma(t)) e^{\beta t} x^{p+\theta}(t) \mathrm{d} B_{2}(t),
\end{aligned}
$$

where $L U: R_{+} \times R_{+} \times[0,+\infty) \times S \longrightarrow R$ is defined by

$$
\begin{aligned}
L U & (x, y, t, i) \\
= & e^{\beta t}\left(\beta+p r(i)+\frac{1}{2} p(p-1) \sigma_{1}^{2}(i)\right) x^{p} \\
& -p a(i) e^{\beta t} x^{\lambda+p}+\frac{1}{2} p(p-1) e^{\beta t} \sigma_{2}^{2}(i) x^{p+2 \theta} \\
& +p b(i) e^{\beta t} x^{p} y^{\lambda} .
\end{aligned}
$$

Applying Young's inequality and (H5), we have

$$
\begin{aligned}
& L U(x, y, t, i) \\
& \leq e^{\beta t}\left(\beta+p r(i)+\frac{1}{2} p(p-1) \sigma_{1}^{2}(i)\right) x^{p} \\
& \quad-p a(i) e^{\beta t} x^{\lambda+p}+\frac{1}{2} p(p-1) e^{\beta t} \sigma_{2}^{2}(i) x^{p+2 \theta} \\
& \quad+p \bar{b} e^{\beta t}\left(\frac{p x^{\lambda+p}}{\lambda+p}+\frac{\lambda y^{\lambda+p}}{\lambda+p}\right) \\
& =e^{\beta t} F_{1}(x, i)+\frac{p \bar{b} \lambda}{\lambda+p} e^{\beta t}\left(-e^{\beta \tau} x^{\lambda+p}+y^{\lambda+p}\right),
\end{aligned}
$$

where

$$
\begin{gathered}
F_{1}(x, i)=\left(\beta+p r(i)+\frac{1}{2} p(p-1) \sigma_{1}^{2}(i)\right) x^{p}+\frac{1}{2} \\
\cdot p(p-1) \sigma_{2}^{2}(i) x^{p+2 \theta}+\left(\frac{\bar{b} p^{2}}{\lambda+p}-p a(i)\right. \\
\left.+\frac{\bar{b} p \lambda}{\lambda+p} e^{\beta \tau}\right) x^{\lambda+p}=(\beta+p r(i) \\
\left.+\frac{1}{2} p(p-1) \sigma_{1}^{2}(i)\right) x^{p}+\left(\frac{1}{2} p(p-1) \sigma_{2}^{2}(i)\right. \\
\left.+\frac{\bar{b} p^{2}}{\lambda+p}-p a(i)+\frac{\bar{b} p \lambda}{\lambda+p} e^{\beta \tau}\right) x^{\lambda+p} .
\end{gathered}
$$

From condition (ii) and $\beta<\min _{i \in S}\{\mu(i)\}$, one can show that

$$
\frac{1}{2} p(p-1) \sigma_{2}^{2}(i)+\frac{\bar{b} p^{2}}{\lambda+p}-p a(i)+\frac{\bar{b} p \lambda}{\lambda+p} e^{\beta \tau}<0,
$$

$i \in S$.

Hence, there exists $M_{2}=M_{2}(p)>0$ such that $F_{1}(x, i) \leq$ $M_{2}, x \in R_{+}, i \in S$. Thus,

$$
\begin{aligned}
L U(x, y, t, i) \leq & M_{2} e^{\beta t} \\
& +\frac{p \bar{b} \lambda}{\lambda+p} e^{\beta t}\left(-e^{\beta \tau} x^{\lambda+p}+y^{\lambda+p}\right) .
\end{aligned}
$$

In addition,

$$
\begin{aligned}
\int_{0}^{t} e^{\beta s} x^{\lambda+p}(s-\tau) \mathrm{d} s \leq & e^{\beta \tau} \int_{-\tau}^{0} \varphi^{\lambda+p}(s) \mathrm{d} s \\
& +e^{\beta \tau} \int_{0}^{t} e^{\beta s} x^{\lambda+p}(s) \mathrm{d} s .
\end{aligned}
$$

From (29), (34), and (35), we have

$$
\begin{aligned}
e^{\beta t} E\left(x^{p}(t)\right) \leq & \varphi^{p}(0)+\frac{M_{2}}{\beta}\left(e^{\beta t}-1\right) \\
& +\frac{p \bar{b} \lambda}{\lambda+p} e^{\beta \tau} \int_{-\tau}^{0} \varphi^{\lambda+p}(s) \mathrm{d} s,
\end{aligned}
$$

which yields

$$
\limsup _{t \rightarrow+\infty} E\left(x^{p}(t)\right) \leq \frac{M_{2}}{\beta}=: K_{1}(p) .
$$

That is, (27) holds. If (i) or (iii) holds, the proof is similar and omitted. Hence, Lemma 8 holds.

Remark 9. If $\lambda=1, \sigma_{2}(i)=0, i \in S$ and (H6) holds, then condition ( $\left.\mathrm{Al}^{\prime}\right)$ in [26] holds. Hence, Lemma 8 generalizes Theorem 3.3 in [26].

Remark 10. If $\lambda>2 \theta, p>0, b(i)=0, i \in S$, then (H6) holds. Hence, Lemma 8 improves and generalizes Lemma 6 in [11].

Theorem 11. Assume that (H7) and (H8) and one of conditions (H4)-(H6) hold. If $0<\theta \leq 1,0<\lambda \leq 1+\theta$ and $b(i) \geq 0, i \in S$, then (6) is stochastically permanent. 
Proof. Clearly, if one of conditions (H4)-(H6) holds, then one of conditions (H1)-(H3) holds. From Theorem 1, for any given $\varphi \in C\left([-\tau, 0], R_{+}\right), \iota \in S$, let $x$ be the solution of the initial value problem (6) and (7). Define

$$
V_{1}(x)=\frac{1}{x^{1+\theta}}, \quad x \in R_{+} .
$$

Using the generalized Itôs formula, we have

$$
\begin{aligned}
& \mathrm{d} V_{1}(x(t))=(1+\theta) V_{1}(x(t))\left[a(\gamma(t)) x^{\lambda}(t)\right. \\
& \left.-b(\gamma(t)) x^{\lambda}(t-\tau)-r(\gamma(t))\right] \mathrm{d} t+\frac{1}{2}(1+\theta)(2 \\
& +\theta) V_{1}(x(t)) \sigma_{1}^{2}(\gamma(t)) \mathrm{d} t+\frac{1}{2}(1+\theta)(2+\theta) \\
& \cdot \sigma_{2}^{2}(\gamma(t)) x^{\theta-1}(t) \mathrm{d} t-(1+\theta) V_{1}(x(t)) \\
& \cdot \sigma_{1}(\gamma(t)) \mathrm{d} B_{1}(t)-(1+\theta) \sigma_{2}(\gamma(t)) \\
& \cdot x^{-1}(t) \mathrm{d} B_{2}(t) .
\end{aligned}
$$

From Lemmas 6 and 7, there is $\alpha>0, w=\left(p_{1}, p_{2}\right.$, $\left.\ldots, p_{m}\right)^{T} \gg 0$ such that $A(\alpha) w \gg 0$. Further, for any $i \in S$,

$$
p_{i}\left[(1+\theta) \alpha \beta(i)-\frac{1}{2}(1+\theta)^{2} \alpha^{2} \sigma_{1}^{2}(i)\right]-\sum_{j=1}^{m} q_{i j} p_{j}
$$

$>0$.

Define $V_{2}(x, i)=p_{i}\left(1+V_{1}(x)\right)^{\alpha}$. By the generalized Itô's formula again,

$$
\begin{aligned}
E V_{2}(x(t), \gamma(t)) & \\
= & V_{2}(\varphi(0), \iota) \\
& +E \int_{0}^{t} L V_{2}(x(s), x(s-\tau), \gamma(s)) \mathrm{d} s
\end{aligned}
$$

where $L V_{2}: R_{+} \times R_{+} \times S \longrightarrow R$ is defined by

$$
\begin{aligned}
& L V_{2}(x, y, i)=\alpha p_{i}(1+\theta)\left(1+V_{1}(x)\right)^{\alpha-2}\{(1 \\
& \left.+V_{1}(x)\right)\left[V_{1}(x)\left(a(i) x^{\lambda}-b(i) y^{\lambda}-r(i)\right)\right. \\
& \left.+\frac{1}{2}(2+\theta) \sigma_{1}^{2}(i) V_{1}(x)+\frac{1}{2}(2+\theta) \sigma_{2}^{2}(i) x^{\theta-1}\right]
\end{aligned}
$$

$$
\begin{aligned}
& +\frac{1}{2}(\alpha-1)(1+\theta) \sigma_{1}^{2}(i) V_{1}^{2}(x)+\frac{1}{2}(\alpha-1)(1+\theta) \\
& \left.\cdot \sigma_{2}^{2}(i) x^{-2}\right\}+\left(1+V_{1}(x)\right)^{\alpha} \sum_{j=1}^{m} q_{i j} p_{j}=(1 \\
& \left.+V_{1}(x)\right)^{\alpha-2}\left\{-\left[p_{i}(1+\theta) \alpha \beta(i)\right.\right.
\end{aligned}
$$$$
\left.-\frac{1}{2} p_{i}(1+\theta)^{2} \alpha^{2} \sigma_{1}^{2}(i)-\sum_{j=1}^{m} q_{i j} p_{j}\right] V_{1}^{2}(x)
$$$$
+\left[-p_{i}(1+\theta) \alpha r(i)+\frac{1}{2} p_{i} \alpha(1+\theta)(2+\theta) \sigma_{1}^{2}(i)\right.
$$$$
\left.+2 \sum_{j=1}^{m} q_{i j} p_{j}\right] V_{1}(x)+\sum_{j=1}^{m} q_{i j} p_{j}+p_{i} \alpha(1+\theta) a(i)
$$$$
\cdot V_{1}(x) x^{\lambda-1-\theta}+p_{i} \alpha(1+\theta) a(i) x^{\lambda-1-\theta}+\frac{1}{2} p_{i} \alpha(1
$$$$
+\theta)(2+\theta) \sigma_{2}^{2}(i) x^{\theta-1}+\frac{1}{2} p_{i} \alpha(\alpha-1)(1+\theta)^{2} \sigma_{2}^{2}(i)
$$$$
\cdot x^{-2}+\frac{1}{2} p_{i} \alpha(1+\theta)(2+\theta) \sigma_{2}^{2}(i) x^{-2}-p_{i} \alpha(1
$$$$
\left.+\theta) b(i) y^{\lambda} V_{1}(x)\left(1+V_{1}(x)\right)\right\} \text {. }
$$

It follows from (40) that there exists $\eta>0$ such that for any $i \in S$,

$$
\begin{aligned}
& p_{i}\left[(1+\theta) \alpha \beta(i)-\frac{1}{2}(1+\theta)^{2} \alpha^{2} \sigma_{1}^{2}(i)\right]-\sum_{j=1}^{m} q_{i j} p_{j} \\
& -\eta p_{i}>0 .
\end{aligned}
$$

From the generalized Itô's formula, we have

$$
\begin{aligned}
& E\left[e^{\eta t} V_{2}(x(t), \gamma(t))\right]=V_{2}(\varphi(0), \iota) \\
& +E \int_{0}^{t}\left[\eta e^{\eta s} V_{2}(x(s), \gamma(s))\right. \\
& \left.+e^{\eta s} L V_{2}(x(s), x(s-\tau), \gamma(s))\right] \mathrm{d} s .
\end{aligned}
$$


In addition,

$$
\begin{aligned}
& \eta e^{\eta t} V_{2}(x, i)+e^{\eta t} L V_{2}(x, y, i)=e^{\eta t}\left(1+V_{1}(x)\right)^{\alpha-2} \\
& \cdot\left[p_{i}(1+\theta) \alpha \beta(i)-\frac{1}{2} p_{i}(1+\theta)^{2} \alpha^{2} \sigma_{1}^{2}(i)\right. \\
& \left.-\sum_{j=1}^{m} q_{i j} p_{j}-\eta p_{i}\right] V_{1}^{2}(x)+\left[-p_{i}(1+\theta) \alpha r(i)\right. \\
& \left.+\frac{1}{2} p_{i} \alpha(1+\theta)(2+\theta) \sigma_{1}^{2}(i)+2 \sum_{j=1}^{m} q_{i j} p_{j}+2 \eta p_{i}\right] \\
& \cdot V_{1}(x)+\eta p_{i}+\sum_{j=1}^{m} q_{i j} p_{j}+p_{i} \alpha(1+\theta) a(i) V_{1}(x) \\
& \cdot x^{\lambda-1-\theta}+p_{i} \alpha(1+\theta) a(i) x^{\lambda-1-\theta}+\frac{1}{2} p_{i} \alpha(1+\theta) \\
& \cdot(2+\theta) \sigma_{2}^{2}(i) x^{\theta-1}+\frac{1}{2} p_{i} \alpha(\alpha-1)(1+\theta)^{2} \sigma_{2}^{2}(i) x^{-2} \\
& +\frac{1}{2} p_{i} \alpha(1+\theta)(2+\theta) \sigma_{2}^{2}(i) x^{-2}-p_{i} \alpha(1+\theta) b(i) \\
& \left.y_{1}(x)\left(1+V_{1}(x)\right)\right\} \leq e^{\eta t} F_{2}(x, i),
\end{aligned}
$$

where

$$
\begin{aligned}
& F_{2}(x, i)=\left(1+V_{1}(x)\right)^{\alpha-2}\left\{-\left[p_{i}(1+\theta) \alpha \beta(i)\right.\right. \\
& \left.+\frac{1}{2} p_{i}(1+\theta)^{2} \alpha^{2} \sigma_{1}^{2}(i)-\sum_{j=1}^{m} q_{i j} p_{j}-\eta p_{i}\right] V_{1}^{2}(x) \\
& +\left[-p_{i}(1+\theta) \alpha \check{r}+\frac{1}{2} p_{i} \alpha(1+\theta)(2+\theta) \widehat{\sigma_{1}^{2}}\right. \\
& \left.+2 \sum_{j=1}^{m} q_{i j} p_{j}+2 \eta p_{i}\right] V_{1}(x)+\eta p_{i}+\sum_{j=1}^{m} q_{i j} p_{j} \\
& +p_{i} \alpha(1+\theta) \widehat{a} V_{1}(x) x^{\lambda-1-\theta}+p_{i} \alpha(1+\theta) \widehat{a} x^{\lambda-1-\theta} \\
& +\frac{1}{2} p_{i} \alpha(2+\theta)^{2} \widehat{\sigma_{2}^{2}} x^{\theta-1}+\frac{1}{2} p_{i} \alpha^{2}(1+\theta)^{2} \widehat{\sigma_{2}^{2}} x^{-2} \\
& \left.+\frac{1}{2} p_{i}(2+\theta)^{2} \widehat{\sigma_{2}^{2}} x^{-2}\right\} \cdot
\end{aligned}
$$

Further,

$$
\lim _{x \rightarrow+\infty} F_{2}(x, i)= \begin{cases}\eta p_{i}+\sum_{j=1}^{m} q_{i j} p_{j}, & \theta<1, \lambda<1+\theta, \\ \eta p_{i}+\sum_{j=1}^{m} q_{i j} p_{j}+\frac{1}{2} p_{i} \alpha(2+\theta)^{2} \widehat{\sigma_{2}^{2}}, & \theta=1, \lambda<1+\theta, \\ \eta p_{i}+\sum_{j=1}^{m} q_{i j} p_{j}+p_{i} \alpha(1+\theta) \widehat{a}, & \theta<1, \lambda=1+\theta, \\ \eta p_{i}+\sum_{j=1}^{m} q_{i j} p_{j}+\frac{1}{2} p_{i} \alpha(2+\theta)^{2} \widehat{\sigma_{2}^{2}}+p_{i} \alpha(1+\theta) \widehat{a}, & \theta=1, \lambda=1+\theta .\end{cases}
$$

Then there is $M_{3}>0$ such that $\sup _{x \in R_{+}, i \in S} F(x, i) \leq M_{3}$. Thus, for any $i \in S$,

$$
\begin{aligned}
p_{i} E & {\left[e^{\eta t}\left(1+V_{1}(x(t))\right)^{\alpha}\right] } \\
& \leq p_{\iota}\left(1+V_{1}(\varphi(0))\right)^{\alpha}+\frac{M_{3}\left(e^{\eta t}-1\right)}{\eta} .
\end{aligned}
$$

Further,

$$
\begin{aligned}
\limsup _{t \rightarrow+\infty} E\left[V_{1}^{\alpha}(x(t))\right] & \leq \limsup _{t \rightarrow+\infty} E\left[\left(1+V_{1}(x(t))\right)^{\alpha}\right] \\
& \leq \frac{M_{3}}{\eta \min \left\{p_{1}, p_{2}, \ldots, p_{m}\right\}} \\
& =: M_{4} .
\end{aligned}
$$

That is, $\lim \sup _{t \rightarrow+\infty} E\left[x^{-\alpha-\alpha \theta}(t)\right] \leq M_{4}$. For any $\varepsilon>0$, let $\delta=\left(\varepsilon / M_{4}\right)^{1 /(\alpha+\alpha \theta)}$. From Chebyshev's inequality,

$$
\begin{aligned}
P\{x(t)<\delta\} & =P\left\{x^{-\alpha-\alpha \theta}(t)>\delta^{-\alpha-\alpha \theta}\right\} \\
& \leq \frac{E\left[x^{-\alpha-\alpha \theta}(t)\right]}{\delta^{-\alpha-\alpha \theta}}=\delta^{\alpha+\alpha \theta} E\left[x^{-\alpha-\alpha \theta}(t)\right] .
\end{aligned}
$$

Further, $\lim \sup _{t \rightarrow+\infty} P\{x(t)<\delta\} \leq \delta^{\alpha+\alpha \theta} M_{4}=\varepsilon$. This yields

$$
\liminf _{t \rightarrow+\infty} P\{x(t) \geq \delta\} \geq 1-\varepsilon
$$

Next we claim that for any $\varepsilon>0$, there exists $H>0$ such that $\liminf \operatorname{int}_{t \rightarrow+\infty} P\{x(t) \leq H\} \geq 1-\varepsilon$. Let $p \in$ $(0,1 / 2)$. From Lemma 8 , there exists $K_{1}(p)>0$ such that $\lim \sup _{t \rightarrow+\infty} E\left(x^{p}(t)\right) \leq K_{1}(p)$. 
For any $\varepsilon>0$, let $H=\left(\varepsilon / K_{1}(p)\right)^{-1 / p}$. Using Chebyshev's inequality yields

$$
P(x(t)>H)=P\left(x^{p}(t)>H^{p}\right) \leq H^{-p} E\left(x^{p}(t)\right),
$$

which means that

$$
\limsup _{t \rightarrow+\infty} P\{x(t)>H\} \leq \limsup _{t \rightarrow+\infty} H^{-p} E\left(x^{p}(t)\right)=\varepsilon .
$$

Hence,

$$
\liminf _{t \longrightarrow+\infty} P\{x(t) \leq H\} \geq 1-\varepsilon .
$$

From (51), (54), and Definition 5, Theorem 11 holds.

\section{Asymptotic Estimation}

In this section, we study some asymptotic properties of (6).

Lemma 12 ((Theorem 2.13, [27]) (BDG's inequality)). Let $g \in$ $\mathfrak{\Omega}^{2}\left(\mathbb{R}_{+} ; \mathbb{R}^{m \times n}\right)$. Define, for $t \geq 0, x(t)=\int_{0}^{t} g(s) d B(s)$ and $A(t)=\int_{0}^{t}|g(s)|^{2} d s$. Then for every $p>0$, there exist universal positive constants $c_{p}, C_{p}$, which are only dependent on $p$, such that

$$
c_{p} E|A(t)|^{p / 2} \leq E\left(\sup _{0 \leq s \leq t}|x(s)|^{p}\right) \leq C_{p} E|A(t)|^{p / 2}
$$

for all $t \geq 0$. In particular, one may take

$$
\begin{aligned}
c_{p} & =\left(\frac{p}{2}\right)^{p}, \\
C_{p} & =\left(\frac{32}{p}\right)^{p / 2},
\end{aligned}
$$$$
\text { if } 0<p<2 \text {; }
$$

$$
c_{p}=1,
$$$$
C_{p}=4 \text {, }
$$

if $p=2$;

$$
\begin{aligned}
c_{p} & =(2 p)^{-p / 2}, \\
C_{p} & =\left[\frac{p^{p+1}}{2(p-1)^{p-1}}\right]^{p / 2},
\end{aligned}
$$

if $p>2$.

Theorem 13. Assume that one of conditions (H4)-(H6) holds. For any $\varphi \in C\left([-\tau, 0] ; R_{+}\right), \iota \in S$, let $x(t)$ be the solution of the initial value problem (6) and (7).

(i) If (H4) holds, then

$$
\limsup _{t \rightarrow+\infty} \frac{\ln x(t)}{\ln t} \leq 2 \quad \text { a.s. }
$$

(ii) If (H5) holds and $\bar{b}<a(i)+(1 / 2)[1-\max \{2,2 \lambda, 1+$ $\lambda\}] \sigma_{2}^{2}(i), i \in S$ or (H6) holds, then

$$
\limsup _{t \rightarrow+\infty} \frac{\ln x(t)}{\ln t} \leq 1 \quad \text { a.s. }
$$

Proof. (i) Assume that (H4) holds. Applying the generalized Itô's formula to $e^{t} \ln x(t)$ yields

$$
\begin{gathered}
e^{t} \ln x(t)-\ln \varphi(0)=\int_{0}^{t} e^{s}[\ln x(s)+\beta(\gamma(s)) \\
-a(\gamma(s)) x^{\lambda}(s)+b(\gamma(s)) x^{\lambda}(s-\tau) \\
\left.-\frac{1}{2} \sigma_{2}^{2}(\gamma(s)) x^{2 \theta}(s)\right] \mathrm{d} s+N_{1}(t)+N_{2}(t),
\end{gathered}
$$

where $N_{1}(t)=\int_{0}^{t} e^{s} \sigma_{1}(\gamma(s)) \mathrm{d} B_{1}(s), N_{2}(t)=$ $\int_{0}^{t} e^{s} \sigma_{2}(\gamma(s)) x^{\theta}(s) \mathrm{d} B_{2}(s)$. For any $\rho>1, v>0$ and $n \in Z^{+}$, it follows from exponential martingale inequality that

$$
\begin{gathered}
P\left\{\sup _{0 \leq t \leq v n}\left\{N_{i}(t)-\frac{1}{2} e^{-v n}\left\langle N_{i}(t), N_{i}(t)\right\rangle\right\}\right. \\
\left.>\rho e^{v n} \ln n\right\} \leq \frac{1}{n^{\rho}}, \quad i=1,2 .
\end{gathered}
$$

From the Borel-Cantelli Lemma [27], for almost all $\omega \in \Omega$, there is a random integer $n_{0}=n_{0}(\omega)$ sufficiently large such that for $n_{0}>n_{0}(\omega), i=1,2$,

$$
N_{i}(t) \leq \frac{1}{2} e^{-v n}\left\langle N_{i}(t), N_{i}(t)\right\rangle+\rho e^{v n} \ln n,
$$

$$
0 \leq t \leq v n \text {. }
$$

This, together with (59), implies that for any $t \in[v(n-$ $3 / 2), v(n-1 / 2)]$ and for almost all $\omega \in \Omega$,

$$
\begin{aligned}
e^{t} \ln x(t) \leq \ln \varphi(0)+\int_{0}^{t} e^{s}[\ln x(s)+\beta(\gamma(s)) \\
-a(\gamma(s)) x^{\lambda}(s)+b(\gamma(s)) x^{\lambda}(s-\tau) \\
+\frac{1}{2} e^{s-v n} \sigma_{1}^{2}(\gamma(s)) \\
\left.-\frac{1}{2}\left(1-e^{s-v n}\right) \sigma_{2}^{2}(\gamma(s)) x^{2 \theta}(s)\right] \mathrm{d} s+2 \rho e^{v n} \\
+\ln n \leq \ln \varphi(0)+\int_{0}^{t} e^{s}[\ln x(s)+\widehat{\beta} \\
-\left(\check{a}-\bar{b} e^{\tau}\right) x^{\lambda}(s)+\frac{1}{2} e^{s-v n} \sigma_{1}^{2}(\gamma(s)) \\
\left.-\frac{1}{2}\left(1-e^{s-v n}\right) \sigma_{2}^{2}(\gamma(s)) x^{2 \theta}(s)\right] \mathrm{d} s \\
+\bar{b} e^{\tau} \int_{-\tau}^{0} \varphi^{\lambda}(s) \mathrm{d} s+2 \rho e^{v n} \ln n \leq \ln \varphi(0) \\
+\int_{0}^{t} e^{s} F_{3}(x(s)) \mathrm{d} s+\bar{b} e^{\tau} \int_{-\tau}^{0} \varphi^{\lambda}(s) \mathrm{d} s+2 \rho e^{v n}
\end{aligned}
$$

$\cdot \ln n$

where $F_{3}(x)=\ln x+\widehat{\beta}-\left(\check{a}-\bar{b} e^{\tau}\right) x^{\lambda}+(1 / 2) e^{-(1 / 2) v} \widehat{\sigma_{1}^{2}}-(1 / 2)(1-$ $\left.e^{-(1 / 2) v}\right) \widehat{\sigma_{2}^{2}} x^{2 \theta}$. Clearly, there exists $M_{5}>0$ such that $F_{3}(x) \leq$ 
$M_{5}$ for any $x \in R_{+}$. Therefore, for almost all $\omega \in \Omega$, if $n \geq$ $n_{0}(\omega), v(n-3 / 2) \leq t \leq v(n-1 / 2)$,

$$
\begin{aligned}
\frac{\ln x(t)}{\ln t} \leq & \frac{\ln \varphi(0)}{e^{t} \ln t}+M_{5} \frac{1-e^{-t}}{\ln t}+\frac{\bar{b} e^{\tau} \int_{-\tau}^{0} \varphi^{\lambda}(s) \mathrm{d} s}{e^{t} \ln t} \\
& +2 \rho e^{-v(n-3 / 2)} e^{v n} \frac{\ln n}{\ln [v(n-3 / 2)]} .
\end{aligned}
$$

This implies

$$
\limsup _{t \rightarrow+\infty} \frac{\ln x(t)}{\ln t} \leq 2 \rho e^{(3 / 2) v}
$$

which is the required assertion (57) by letting $\rho \longrightarrow 1$ and $v \longrightarrow 0$.

(ii) Assume that (H5) and $\bar{b}<a(i)+(1 / 2)[1-$ $\max \{2,2 \lambda, 1+\lambda\}] \sigma_{2}^{2}(i), i \in S$ or (H6) holds. It follows from Theorem 1 that for any $t \geq \tau, x(t) \in R_{+}$a.s. This, together with (5), yields

$$
\begin{aligned}
\mathrm{d} x(t) \leq & \left(\widehat{r} x(t)+\bar{b} x(t) x^{\lambda}(t-\tau)\right) \mathrm{d} t \\
& +\sigma_{1}(\gamma(t)) x(t) \mathrm{d} B_{1}(t) \\
& +\sigma_{2}(\gamma(t)) x^{1+\theta}(t) \mathrm{d} B_{2}(t) .
\end{aligned}
$$

Further,

$$
\begin{aligned}
& E\left\{\sup _{t \leq u \leq t+1} x(u)\right\} \\
& \leq E(x(t))+\widehat{r} \int_{t}^{t+1} E(x(s)) \mathrm{d} s \\
&+\bar{b} \int_{t}^{t+1} E\left(x(s) x^{\lambda}(s-\tau)\right) \mathrm{d} s \\
&+E\left\{\sup _{t \leq u \leq t+1} \int_{t}^{u} \sigma_{1}(\gamma(s)) x(s) \mathrm{d} B_{1}(s)\right\} \\
&+E\left\{\sup _{t \leq u \leq t+1} \int_{t}^{u} \sigma_{2}(\gamma(s)) x^{1+\theta}(s) \mathrm{d} B_{2}(s)\right\} .
\end{aligned}
$$

Using the BDG's inequality, there exist $C_{1}>0$ and $C_{2}>0$ such that

$$
\begin{aligned}
& E\left\{\sup _{t \leq u \leq t+1} \int_{t}^{u} \sigma_{1}(\gamma(s)) x(s) \mathrm{d} B_{1}(s)\right\} \\
& \leq C_{1} E\left\{\int_{t}^{t+1}\left[\sigma_{1}(\gamma(s)) x(s)\right]^{2} \mathrm{~d} s\right\}^{1 / 2} \\
& \quad \leq E\left\{\frac{1}{2} \sup _{t \leq u \leq t+1} x(u) \cdot 2 C_{1}^{2} \bar{\sigma}_{1}^{2} \int_{t}^{t+1} x(s) \mathrm{d} s\right\}^{1 / 2} \\
& \quad \leq \frac{1}{4} E\left(\sup _{t \leq u \leq t+1} x(u)\right)+C_{1}^{2}{\overline{\sigma_{1}}}^{2} \int_{t}^{t+1} E(x(s)) \mathrm{d} s,
\end{aligned}
$$

and

$$
\begin{aligned}
E\left\{\sup _{t \leq u \leq t+1} \int_{t}^{u} \sigma_{2}(\gamma(s)) x^{1+\theta}(s) \mathrm{d} B_{2}(s)\right\} \\
\leq C_{2} E\left\{\int_{t}^{t+1}\left[\sigma_{2}(\gamma(s)) x^{1+\theta}(s)\right]^{2} \mathrm{~d} s\right\}^{1 / 2} \\
\leq E\left\{\frac{1}{2} \sup _{t \leq u \leq t+1} x(u) \cdot 2 C_{2}^{2}{\overline{\sigma_{2}}}^{2} \int_{t}^{t+1} x^{1+2 \theta}(s) \mathrm{d} s\right\}^{1 / 2} \\
\leq \frac{1}{4} E\left(\sup _{t \leq u \leq t+1} x(u)\right) \\
+C_{2}^{2}{\overline{\sigma_{2}}}^{2} \int_{t}^{t+1} E\left(x^{1+2 \theta}(s)\right) \mathrm{d} s .
\end{aligned}
$$

From Hölder's inequality,

$$
\begin{aligned}
& \int_{t}^{t+1} E\left(x(s) x^{\lambda}(s-\tau)\right) \mathrm{d} s \\
& \quad \leq \int_{t}^{t+1}\left(E\left(x^{2}(s)\right)\right)^{1 / 2}\left(E\left(x^{2 \lambda}(s-\tau)\right)\right)^{1 / 2} \mathrm{~d} s \\
& \quad \leq \frac{1}{2} \int_{t}^{t+1} E\left(x^{2}(s)\right) \mathrm{d} s+\frac{1}{2} \int_{t}^{t+1} E\left(x^{2 \lambda}(s-\tau)\right) \mathrm{d} s .
\end{aligned}
$$

Hence,

$$
\begin{aligned}
E\left\{\sup _{t \leq u \leq t+1} x(u)\right\} \leq & 2 E(x(t))+2 \widehat{r} \int_{t}^{t+1} E(x(s)) \mathrm{d} s \\
& +\bar{b} \int_{t}^{t+1} E\left(x^{2}(s) \mathrm{d} s\right. \\
& +\bar{b} \int_{t}^{t+1} E\left(x^{2 \lambda}(s-\tau) \mathrm{d} s\right. \\
& +2 C_{1}^{2}{\overline{\sigma_{1}}}^{2} \int_{t}^{t+1} E(x(s)) \mathrm{d} s \\
& +2 C_{2}^{2}{\overline{\sigma_{2}}}^{2} \int_{t}^{t+1} E\left(x^{1+2 \theta}(s)\right) \mathrm{d} s .
\end{aligned}
$$

It follows from (70) and Lemma 8 that

$$
\begin{aligned}
\limsup _{t \rightarrow+\infty} E\left(\sup _{t \leq u \leq t+1} x(u)\right) \\
\leq\left(2+2 \widehat{r}+2 C_{1}^{2}{\overline{\sigma_{1}}}^{2}\right) K_{1}(1)+\bar{b}\left(K_{1}(2)+K_{1}(2 \lambda)\right) \\
\quad+2 C_{2}^{2}{\overline{\sigma_{2}}}^{2} K_{1}(1+2 \theta) .
\end{aligned}
$$

From (71), there is $M_{6}>0$ such that

$$
E\left(\sup _{k \leq t \leq k+1} x(t)\right) \leq M_{6}, \quad k=1,2, \cdots .
$$

For any $\varepsilon>0$, from Chebyshev's inequality, we have

$$
P\left(\sup _{k \leq t \leq k+1} x(t)>k^{1+\varepsilon}\right) \leq \frac{M_{6}}{k^{1+\varepsilon}}, \quad k=1,2, \cdots .
$$


From the Borel-Cantelli Lemma [27], for almost all $\omega \in \Omega$, there exists an integer $k_{0}(\omega)$ such that for any $k \geq k_{0}(\omega)$,

$$
\sup _{k \leq t \leq k+1} x(t, \omega) \leq k^{1+\varepsilon}
$$

Consequently, for any $\varepsilon>0$ and almost all $\omega \in \Omega$, if $k \geq k_{0}(\omega)$ and $k \leq t \leq k+1$, then

$$
\frac{\ln x(t, \omega)}{\ln t} \leq \frac{(1+\varepsilon) \ln k}{\ln k}=1+\varepsilon
$$

Therefore

$$
\limsup _{t \rightarrow+\infty} \frac{\ln x(t)}{\ln t} \leq 1+\varepsilon \quad \text { a.s. }
$$

Letting $\varepsilon \longrightarrow 0$, we obtain the desired assertion (58). hold.

From Theorem 13, it is easy to see that the following results

Corollary 14. Assume that $b(i)=0, i \in S$. For any $x_{0}>0, \iota \in$ $S$, let $x(t)$ be the solution of (3) with the initial condition $x(0)=$ $x_{0}$ and $\gamma(0)=\imath$. (i) If $\lambda<2 \theta$, then (57) holds; (ii) if $\lambda \geq 2 \theta$, then (58) holds.

Corollary 15. Assume that $\lambda=1, \theta=1 / 4, \sigma_{2}(i)=0, i \in S$. For any $\varphi \in C\left([-\tau, 0] ; R_{+}\right)$, let $x(t)$ be the solution of the initial value problem (6) and (7). If $\bar{b}<\check{a}$, then (58) holds.

Remark 16. Theorem 6 in [12] shows that (57) holds for any $\lambda>0, \theta>0$. Hence, Corollary 14 improves Theorem 6 in [12]. In addition, Corollary 15 is corresponding to Lemma 5.1 in [26]. Hence, Theorem 13 generalizes and improves Theorem 6 in [12] and Lemma 5.1 in [26].

Theorem 17. Assume that (H7), (H8), and one of conditions (H4)-(H6) hold. For any $\varphi \in C\left([-\tau, 0] ; R_{+}\right), \iota \in S$, let $x(t)$ be the solution of the initial value problem (6) and (7). If $0<\theta \leq$ $1,0<\lambda \leq 1+\theta$ and $b(i) \geq 0, i \in S$, then

$$
\liminf _{t \rightarrow+\infty} \frac{\ln x(t)}{\ln t} \geq-\frac{1}{(1+\theta) \alpha} \quad \text { a.s. }
$$

Proof. From (39) and the generalized Itô's formula,

$$
\begin{aligned}
\mathrm{d}[ & \left.\left(1+V_{1}(x(t))\right)^{\alpha}\right] \leq \alpha(1+\theta)\left(1+V_{1}(x(t))\right)^{\alpha-2} \\
& \cdot\left\{\left[-\check{\beta}+\frac{1}{2} \alpha(1+\theta) \bar{\sigma}_{1}^{2}+1\right] V_{1}^{2}(x(t))+F_{4}(x)\right\} \\
& -\alpha(1+\theta)\left(1+V_{1}(x(t))\right)^{\alpha-1} \\
& \cdot\left[\sigma_{1}(\gamma(t)) V_{1}(x(t)) \mathrm{d} B_{1}(t)\right. \\
& \left.+\sigma_{2}(\gamma(t)) x^{-1}(t) \mathrm{d} B_{2}(t)\right],
\end{aligned}
$$

where

$$
\begin{aligned}
F_{4}(x)= & -V_{1}^{2}(x)+\left[-\check{r}+\frac{1}{2}(1+\theta) \bar{\sigma}_{1}^{2}\right] V_{1}(x) \\
& +\widehat{a} V_{1}(x) x^{\lambda-1-\theta}+\widehat{a} x^{\lambda-1-\theta} \\
& +\frac{1}{2}(2+\theta){\overline{\sigma_{2}}}^{2} x^{\theta-1}+\frac{1}{2} \alpha(1+\theta){\overline{\sigma_{2}}}^{2} x^{-2} \\
& +\frac{1}{2}(1+\theta){\overline{\sigma_{2}}}^{2} x^{-2} .
\end{aligned}
$$

Clearly, there exists $M_{7}>0$ such that $F_{4}(x) \leq M_{7}$ for any $x \in(0,+\infty)$. Further,

$$
\begin{aligned}
& \mathrm{d}\left[\left(1+V_{1}(x(t))\right)^{\alpha}\right] \leq \alpha(1+\theta)\left(1+V_{1}(x(t))\right)^{\alpha-2} \\
& \cdot\left\{\left[-\check{\beta}+\frac{1}{2} \alpha(1+\theta){\overline{\sigma_{1}}}^{2}+1\right] V_{1}^{2}(x(t))+M_{7}\right\} \mathrm{d} t \\
& \quad-\alpha(1+\theta)\left(1+V_{1}(x(t))\right)^{\alpha-1} \\
& \cdot\left[\sigma_{1}(\gamma(t)) V_{1}(x(t)) \mathrm{d} B_{1}(t)\right. \\
& \left.+\sigma_{2}(\gamma(t)) x^{-1}(t) \mathrm{d} B_{2}(t)\right] \leq \alpha(1+\theta) M_{8}(1 \\
& \left.+V_{1}(x(t))\right)^{\alpha} \mathrm{d} t-\alpha(1+\theta)\left(1+V_{1}(x(t))\right)^{\alpha-1} \\
& \quad \cdot\left[\sigma_{1}(\gamma(t)) V_{1}(x(t)) \mathrm{d} B_{1}(t)\right. \\
& \left.+\sigma_{2}(\gamma(t)) x^{-1}(t) \mathrm{d} B_{2}(t)\right]
\end{aligned}
$$

where $M_{8}=\max \left\{-\check{\beta}+(1 / 2) \alpha(1+\theta){\overline{\sigma_{1}}}^{2}+1, M_{7}\right\}$ are positive numbers. By (49), there exists $M_{9}>0$ such that

$$
E\left[\left(1+V_{1}(x(t))\right)^{\alpha}\right] \leq M_{9}, \quad t \geq 0 .
$$

Let $\delta>0$ satisfy

$$
\alpha(1+\theta)\left[M_{8} \delta+6 \sqrt{\delta}\left(\bar{\sigma}_{1}+\bar{\sigma}_{2}\right)\right]<\frac{1}{2}
$$

From (80), for any $k \in\{1,2, \cdots\}$,

$$
\begin{aligned}
& E\left\{\sup _{(k-1) \delta \leq t \leq k \delta}\left(1+V_{1}(x(t))\right)^{\alpha}\right\} \\
& \quad \leq E\left\{\left[1+V_{1}(x(k-1))\right.\right. \\
& \left.\quad \cdot \delta]^{\alpha}\right\} \\
& \quad+\alpha(1+\theta) M_{8} E\left\{\sup _{(k-1) \delta \leq t \leq k \delta} \int_{(k-1) \delta}^{t}\left(1+V_{1}(x(s))\right)^{\alpha} \mathrm{d} s\right\} \\
& \quad+\alpha(1+\theta) E\left\{\sup _{(k-1) \delta \leq t \leq k \delta}\left|\int_{(k-1) \delta}^{t}\left(1+V_{1}(x(s))\right)^{\alpha-1} \sigma_{1}(\gamma(s)) V_{1}(x(s)) \mathrm{d} B_{1}(s)\right|\right\} \\
& \quad+\alpha(1+\theta) E\left\{\sup _{(k-1) \delta s t \leq k \delta} \mid \int_{(k-1) \delta}^{t}\left(1+V_{1}(x(s))\right)^{\alpha-1} \sigma_{2}(\gamma(s))\right. \\
& \left.\quad \cdot x^{-1}(s) \mathrm{d} B_{2}(s) \mid\right\} .
\end{aligned}
$$


In addition, we have

$$
\begin{gathered}
E\left\{\sup _{(k-1) \delta \leq t \leq k \delta} \int_{(k-1) \delta}^{t}\left(1+V_{1}(x(s))\right)^{\alpha} \mathrm{d} s\right\} \\
\leq E \int_{(k-1) \delta}^{k \delta}\left(1+V_{1}(x(s))\right)^{\alpha} \mathrm{d} s \\
\leq \delta E\left\{\sup _{(k-1) \delta \leq t \leq k \delta}\left(1+V_{1}(x(t))\right)^{\alpha}\right\} .
\end{gathered}
$$

From the BDG's inequality,

$$
\begin{gathered}
E\left\{\sup _{(k-1) \delta \leq t \leq k \delta}\left|\int_{(k-1) \delta}^{t}\left(1+V_{1}(x(s))\right)^{\alpha-1} \sigma_{1}(\gamma(s)) V_{1}(x(s)) \mathrm{d} B_{1}(s)\right|\right\} \\
\leq 6 E\left\{\int_{(k-1) \delta}^{k \delta}\left(1+V_{1}(x(s))\right)^{2 \alpha-2} \sigma_{1}^{2}(\gamma(s)) V_{1}^{2}(x(s)) \mathrm{d} s\right\}^{1 / 2} \\
\leq 6 \bar{\sigma}_{1} E\left\{\int_{(k-1) \delta}^{k \delta}\left(1+V_{1}(x(s))\right)^{2 \alpha} \mathrm{d} s\right\}^{1 / 2} \\
\leq 6 \sqrt{\delta} \bar{\sigma}_{1} E\left\{\sup _{(k-1) \delta \leq t \leq k \delta}\left(1+V_{1}(x(t))\right)^{\alpha}\right\},
\end{gathered}
$$

and

$$
\begin{gathered}
E\left\{\sup _{(k-1) \delta \leq t \leq k \delta}\left|\int_{(k-1) \delta}^{t}\left(1+V_{1}(x(s))\right)^{\alpha-1} \sigma_{2}(\gamma(s)) x^{-1}(s) \mathrm{d} B_{2}(s)\right|\right\} \\
\leq 6 E\left\{\int_{(k-1) \delta}^{k \delta}\left(1+V_{1}(x(s))\right)^{2 \alpha-2} \sigma_{2}^{2}(\gamma(s)) x^{-2}(s) \mathrm{d} s\right\}^{1 / 2} \\
\leq 6 \bar{\sigma}_{2} E\left\{\int_{(k-1) \delta}^{k \delta}\left(1+V_{1}(x(s))\right)^{2 \alpha} \mathrm{d} s\right\}^{1 / 2} \\
\leq 6 \sqrt{\delta} \bar{\sigma}_{2} E\left\{\sup _{(k-1) \delta \leq t \leq k \delta}\left(1+V_{1}(x(t))\right)^{\alpha}\right\} .
\end{gathered}
$$

From (83)-(86),

$$
\begin{aligned}
E\left\{\sup _{(k-1) \delta \leq t \leq k \delta}\left(1+V_{1}(x(t))\right)^{\alpha}\right\} \\
\leq E\left\{\left[1+V_{1}(x(k-1)) \delta\right]^{\alpha}\right\}+\alpha(1+\theta) \\
\cdot M_{8} \delta E\left\{\sup _{(k-1) \delta \leq t \leq k \delta}\left(1+V_{1}(x(t))\right)^{\alpha}\right\}+6 \alpha(1+\theta) \\
\cdot \sqrt{\delta}\left(\bar{\sigma}_{1}+\bar{\sigma}_{2}\right) E\left\{\sup _{(k-1) \delta \leq t \leq k \delta}\left(1+V_{1}(x(t))\right)^{\alpha}\right\} \\
=E\left\{\left(1+V_{1}(x(k-1)) \delta\right)^{\alpha}\right\}+\alpha(1+\theta) \\
\cdot\left[M_{8} \delta+6 \sqrt{\delta}\left(\bar{\sigma}_{1}+\bar{\sigma}_{2}\right)\right] \\
\cdot E\left\{\sup _{(k-1) \delta \leq t \leq k \delta}\left(1+V_{1}(x(t))\right)^{\alpha}\right\} .
\end{aligned}
$$

This, together with (81) and (82), yields

$$
E\left\{\sup _{(k-1) \delta \leq t \leq k \delta}\left(1+V_{1}(x(t))\right)^{\alpha}\right\} \leq 2 M_{9} .
$$

For any $\varepsilon>0$, using Chebyshev's inequality,

$$
P\left\{\sup _{(k-1) \delta \leq t \leq k \delta}\left(1+V_{1}(x(t))\right)^{\alpha}>(k \delta)^{1+\varepsilon}\right\} \leq \frac{2 M_{9}}{(k \delta)^{1+\varepsilon}} .
$$

From the Borel-Cantelli Lemma, for almost all $\omega \in \Omega$, there exists $k_{0}(\omega)>0$ such that for any $k>k_{0}(\omega)$,

$$
\sup _{(k-1) \delta \leq t \leq k \delta}\left(1+V_{1}(x(t, \omega))\right)^{\alpha} \leq(k \delta)^{1+\varepsilon},
$$

which yields,

$$
\frac{\ln \left(1+V_{1}(x(t))\right)^{\alpha}}{\ln t} \leq \frac{(1+\varepsilon) \ln (k \delta)}{\ln [(k-1) \delta]}<1+\varepsilon \quad \text { a.s. }
$$

Therefore

$$
\limsup _{t \rightarrow+\infty} \frac{\ln \left(1+V_{1}(x(t))\right)^{\alpha}}{\ln t} \leq 1+\varepsilon \quad \text { a.s. }
$$

Letting $\varepsilon \quad \longrightarrow \quad 0$, we have $\lim \sup _{t \rightarrow+\infty}(\ln (1+$ $\left.\left.V_{1}(x(t))\right)^{\alpha} / \ln t\right) \leq 1$ a.s. Further,

$$
\limsup _{t \rightarrow+\infty} \frac{\ln (x(t))^{-(1+\theta) \alpha}}{\ln t} \leq 1 \quad \text { a.s. }
$$

Hence,

$$
\begin{aligned}
& \limsup _{t \rightarrow+\infty} \frac{\ln (x(t))^{-(1+\theta) \alpha}}{\ln t} \\
& \quad=-(1+\theta) \alpha \liminf _{t \rightarrow+\infty} \frac{\ln (x(t))}{\ln t} \leq 1 \quad \text { a.s. }
\end{aligned}
$$

This yields (77). Hence, Theorem 17 holds.

Theorem 18. Assume that the conditions of Theorem 13 hold. If (H7)-(H9) hold, then for any $\varphi \in C\left([-\tau, 0] ; R_{+}\right), \iota \in S$, the solution $x(t)$ of (6) obeys

$$
\limsup _{t \rightarrow+\infty} \frac{1}{t} \int_{0}^{t} x^{\lambda}(s) \mathrm{d} s \leq \frac{1}{\check{a}-\bar{b}} \sum_{i=1}^{m} \pi_{i} \beta(i) \quad \text { a.s. }
$$

Proof. From Theorems 13 and 17,

$$
\lim _{t \rightarrow+\infty} \frac{\ln x(t)}{t}=0 \text { a.s. }
$$

Using the generalized Itô's formula, we obtain

$$
\begin{aligned}
\ln x(t)= & \ln x_{0}+\int_{0}^{t}\left[r(\gamma(s))-\frac{1}{2} \sigma_{1}^{2}(\gamma(s))\right] \mathrm{d} s \\
& -\int_{0}^{t} a(\gamma(s)) x^{\lambda}(s) \mathrm{d} s \\
& +\int_{0}^{t} b(\gamma(s)) x^{\lambda}(s-\tau) \mathrm{d} s \\
& -\frac{1}{2} \int_{0}^{t} \sigma_{2}^{2}(\gamma(s)) x^{2 \theta}(s) \mathrm{d} s+M_{1}(t) \\
& +M_{2}(t),
\end{aligned}
$$


where $M_{1}(t)=\int_{0}^{t} \sigma_{1}\left(\gamma(s) \mathrm{d} B_{1}(s), M_{2}(t)=\int_{0}^{t} \sigma_{2}\left(\gamma(s) x^{\theta}(s) \mathrm{d} B_{2}(s)\right.\right.$. The quadratic variation of $M_{2}(t)$ is

$$
\left\langle M_{2}(t), M_{2}(t)\right\rangle=\int_{0}^{t} \sigma_{2}^{2}(\gamma(s)) x^{2 \theta}(s) \mathrm{d} s .
$$

From the exponential martingale inequality, for any integer $n \geq 2$,

$$
\begin{aligned}
& P\left\{\sup _{0 \leq t \leq n}\left\{M_{2}(t)-\frac{1}{2}\left\langle M_{2}(t), M_{2}(t)\right\rangle\right\}>2 \ln n\right\} \\
& \leq e^{-2 \ln n}=\frac{1}{n^{2}} .
\end{aligned}
$$

From Borel-Cantelli Lemma, for almost all $\omega \in \Omega$, there exists a random integer $n_{0}$ such that for $n \geq n_{0}(\omega)$,

$$
\sup _{0 \leq t \leq n}\left\{M_{2}(t)-\frac{1}{2}\left\langle M_{2}(t), M_{2}(t)\right\rangle\right\} \leq 2 \ln n .
$$

Further, for almost all $\omega \in \Omega, n \geq n_{0}(\omega), 0 \leq t \leq n$,

$$
\begin{aligned}
M_{2}(t) & \leq 2 \ln n+\frac{1}{2}\left\langle M_{2}(t), M_{2}(t)\right\rangle \\
& =2 \ln n+\frac{1}{2} \int_{0}^{t} \sigma_{2}^{2}(\gamma(s)) x^{2 \theta}(s) \mathrm{d} s .
\end{aligned}
$$

Note that

$$
\begin{aligned}
& \int_{0}^{t}\left.\left.b(\gamma(s)) x^{\lambda}(s-\tau)\right) \mathrm{d} s \leq \bar{b} \int_{0}^{t} x^{\lambda}(s-\tau)\right) \mathrm{d} s \\
&= \bar{b} \int_{-\tau}^{t-\tau} x^{\lambda}(s) \mathrm{d} s \\
& \leq \bar{b} \int_{-\tau}^{0} x^{\lambda}(s) \mathrm{d} s+\bar{b} \int_{0}^{t} x^{\lambda}(s) \mathrm{d} s .
\end{aligned}
$$

From (97), for any $n \geq n_{0}, t \in(n-1, n]$

$$
\begin{aligned}
\frac{\ln x(t)}{t} \leq & \frac{\ln x_{0}}{t}+\frac{1}{t} \int_{0}^{t} \beta(\gamma(s)) \mathrm{d} s \\
& +\frac{-\check{a}+\bar{b}}{t} \int_{0}^{t} x^{\lambda}(s) \mathrm{d} s+\frac{\bar{b}}{t} \int_{-\tau}^{0} x^{\lambda}(s) \mathrm{d} s \\
& +\frac{2 \ln n}{n-1}+\frac{M_{1}(t)}{t} .
\end{aligned}
$$

Let $t \longrightarrow+\infty$, using the strong law of large numbers for martingales and (96), we obtain

$$
\limsup _{t \rightarrow+\infty} \frac{1}{t} \int_{0}^{t} x^{\lambda}(s) \mathrm{d} s \leq \frac{1}{\check{a}-\bar{b}} \sum_{i=1}^{m} \pi_{i} \beta(i) \quad \text { a.s. }
$$

We then obtain assertions (95). Hence, Theorem 18 holds.

Remark 19. Liu et al. [12] have claimed that, under (H7) and (H8), the solution of (2) obeys

$$
\limsup _{t \rightarrow+\infty} \frac{1}{t} \int_{0}^{t} x^{\lambda}(s) \mathrm{d} s \leq \frac{1}{\check{a}} \sum_{i=1}^{m} \pi_{i} \beta(i) \quad \text { a.s. }
$$

It is easy to see that if $b(i)=0, i \in S$, then (95) becomes (105). Hence, Theorem 18 generalizes Theorem 8 in [12].
Remark 20. If $\lambda=1, \sigma_{2}(i)=0, i \in S$, then conditions (H7)(H9) are corresponding to the conditions (A1"), (A2), and (A3) in [26]. Hence, Theorem 18 generalizes Theorem 5.3 in [26].

\section{Numerical Simulations}

In this section, we make numerical simulations to illustrate our theoretical results. Consider the following examples.

Example 1. In (6), let $\lambda=2, \theta=1, \tau=1, S=\{1,2\}$, $q_{12}=-q_{11}=1, q_{21}=-q_{22}=2, r(1)=3, a(1)=4, b(1)=$ $2, \sigma_{1}(1)=0.2, \sigma_{2}(1)=0.2, r(2)=1, a(2)=3, b(2)=$ $1.5, \sigma_{1}(2)=0.15, \sigma_{2}(2)=0.2$ and initial value $x(s)=1.5, s \in$ $[-1,0], \gamma(0)=1$.

Clearly, $\check{a}=3, \bar{b}=2, \beta(1)=2.98, \beta(2) \approx 0.989, \pi_{1}=$ $2 / 3, \pi_{2}=1 / 3, \pi_{1} \beta(1)+\pi_{2} \beta(2) \approx 2.316>0$. Hence, $(\mathrm{H} 5)$ and (H7)-(H9) hold. Further, all conditions of Theorems 11 and 18 are satisfied. According to Theorems 11 and 18, (6) is stochastically permanent and its solution $x(t)$ with any positive initial value satisfies

$$
\underset{t \rightarrow+\infty}{\limsup } \frac{1}{t} \int_{0}^{t} x^{2}(s) \mathrm{d} s \leq 2.316 \quad \text { a.s. }
$$

In Figure 1, (i) left panel shows stochastic trajectories of model (6) with parameters in Example 1; deterministic trajectory of model (6) with $r(i)=3, a(i)=4, b(i)=$ $2, \sigma_{1}(i)=0, \sigma_{2}(i)=0, \lambda=2, \tau=1, i=1,2$; (ii) right panel shows stochastic trajectories of model (6) with parameters in Example 1; deterministic trajectory of model (6) with $r(i)=$ $1, a(i)=3, b(i)=1.5, \sigma_{1}(i)=0, \sigma_{2}(i)=0, \lambda=2, \tau=1, i=$ 1,2 . Figure 1 clearly supports Theorems 11 and 18 .

Example 2. In (6), let $\lambda=1.5, \theta=0.7, \tau=1, S=\{1,2\}$, $q_{11}=q_{22}=-1, q_{12}=q_{21}=1, r(1)=0.4, a(1)=0.15, b(1)=$ $0.1, \sigma_{1}(1)=0.03, \sigma_{2}(1)=0.013, r(2)=0.3, a(2)=0.2, b(2)=$ $0.09, \sigma_{1}(2)=0.02, \sigma_{2}(2)=0.021$ and initial value $x(s)=$ $1, s \in[-1,0], \gamma(0)=2$.

Clearly, $\check{a}=0.15, \bar{b}=0.1, \beta(1)=0.39955, \beta(2)=$ $0.2998, \pi_{1}=1 / 2, \pi_{2}=1 / 2, \pi_{1} \beta(1)+\pi_{2} \beta(2) \approx 0.3497>0$. Hence, (H6)-(H9) hold. Further, all conditions of Theorems 11 and 18 are satisfied. From Theorems 11 and 18, (6) is stochastically permanent and its solution $x(t)$ with any positive initial value satisfies

$$
\underset{t \rightarrow+\infty}{\limsup } \frac{1}{t} \int_{0}^{t} x^{1.5}(s) \mathrm{d} s \leq 6.994 \text { a.s. }
$$

In Figure 2, (i) left panel shows stochastic trajectories of model (6) with parameters in Example 2; deterministic trajectory of model (6) with $r(i)=0.4, a(i)=0.15, b(i)=$ $0.1, \sigma_{1}(i)=0, \sigma_{2}(i)=0, \lambda=1.5, \tau=1, i=1,2$; (ii) right panel shows stochastic trajectories of model (6) with parameters in Example 2; deterministic trajectory of model (6) with $r(i)=0.3, a(i)=0.2, b(i)=0.09, \sigma_{1}(i)=0, \sigma_{2}(i)=$ $0, \lambda=2, \tau=1, i=1,2$. Figure 2 clearly supports Theorems 11 and 18 . 


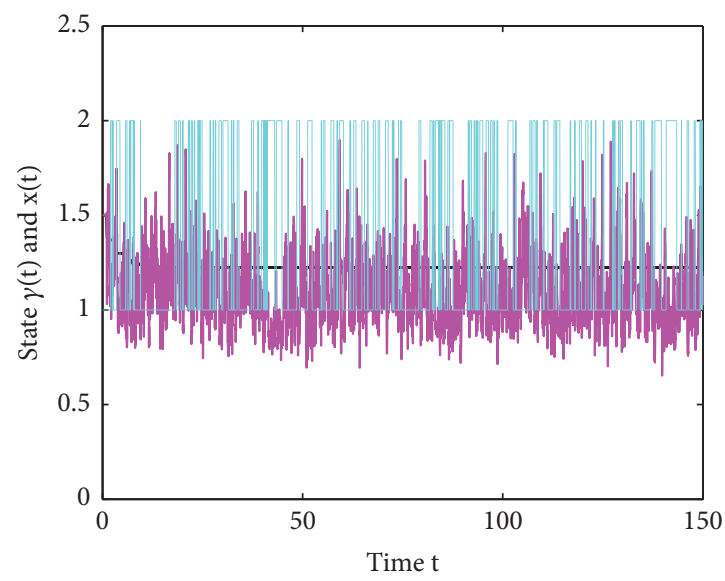

Deterministic solution $\mathrm{x}(\mathrm{t})$
Stochastic solution $\mathrm{x}(\mathrm{t})$
Markov chain $\gamma(\mathrm{t})$

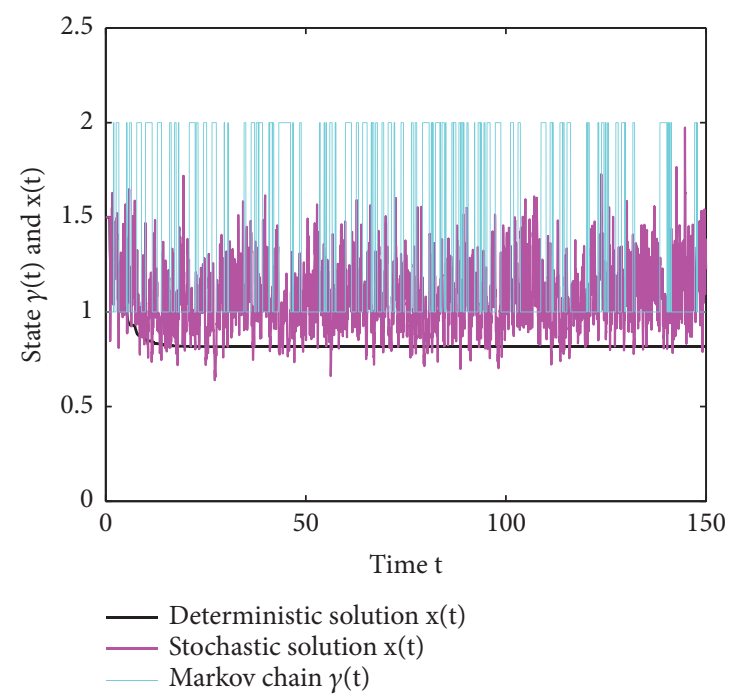

FIgURE 1: Stochastic and deterministic trajectories of model (6).
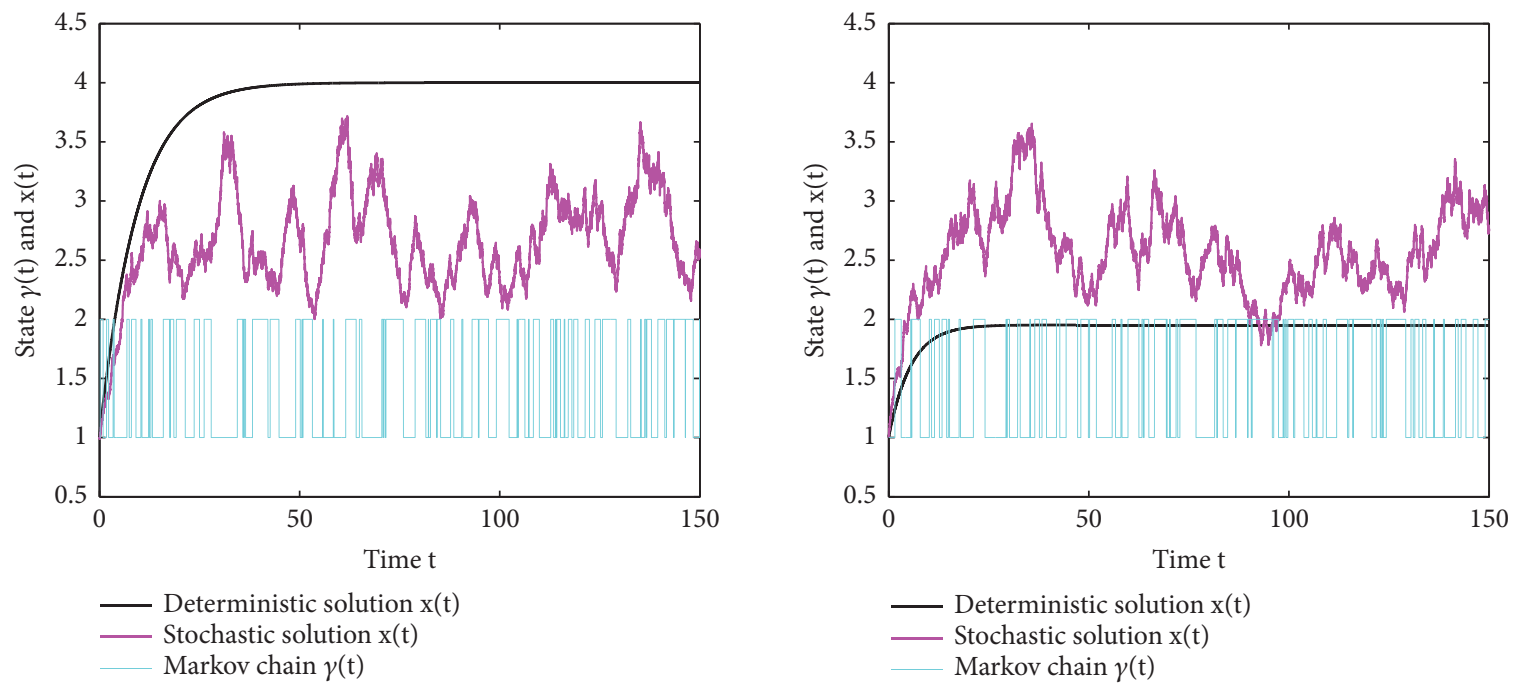

FIgURE 2: Stochastic and deterministic trajectories of model (6).

Example 3. In (6), let $\lambda=1, \theta=0.25, \tau=1, S=\{1,2\}$, $q_{12}=-q_{11}=1, q_{21}=-q_{22}=2, r(1)=0.2, a(1)=0.5, b(1)=$ $0.3, \sigma_{1}(1)=0.4, \sigma_{2}(1)=0.1, r(2)=0, a(2)=0.4, b(2)=$ $0.2, \sigma_{1}(2)=0.5, \sigma_{2}(2)=0$ and initial value $x(s)=1.5, s \in$ $[-1,0], \gamma(0)=1$.

Clearly, $\check{a}=0.4, \bar{b}=0.3, \beta(1)=0.12, \beta(2)=-0.125, \pi_{1}=$ $2 / 3, \pi_{2}=1 / 3, \pi_{1} \beta(1)+\pi_{2} \beta(2) \approx 0.0383>0$. Hence, $(\mathrm{H} 6)$ (H8) hold. Hence, all conditions of Theorem 11 are satisfied. According to Theorem 11, (6) is stochastically permanent and its solution $x(t)$ with any positive initial value satisfies

$$
\limsup _{t \rightarrow+\infty} \frac{1}{t} \int_{0}^{t} x(s) \mathrm{d} s \leq 0.383 \text { a.s. }
$$

Figure 3 clearly supports Theorems 11 and 18.
Assume that the Markov chain $\gamma(t)=1 \in S$. Then (6) obeys the following subsystem:

$$
\begin{aligned}
\mathrm{d} x(t)= & x(t)[0.2-0.5 x(t)+0.3 x(t-1)] \mathrm{d} t \\
& +0.4 x(t) \mathrm{d} B_{1}(t)+0.1 x^{1.25}(t) \mathrm{d} B_{2}(t) .
\end{aligned}
$$

Similarly, assume that the Markov chain $\gamma(t)=2 \in S$. Then (6) obeys the following subsystem:

$$
\begin{aligned}
\mathrm{d} x(t)= & x(t)[-0.4 x(t)+0.2 x(t-1)] \mathrm{d} t \\
& +0.5 x(t) \mathrm{d} B_{1}(t)
\end{aligned}
$$

For subsystem (109), it is easy to check that all conditions of Theorem 11 are satisfied. Hence, subsystem (109) is stochastically permanent. In addition, for subsystem (110), $-a(2)+$ $|b(2)|=-0.4+0.2=-0.2<0, r(2)-0.5 \sigma_{1}^{2}(2)=-0.5 \times 0.5^{2}=$ 


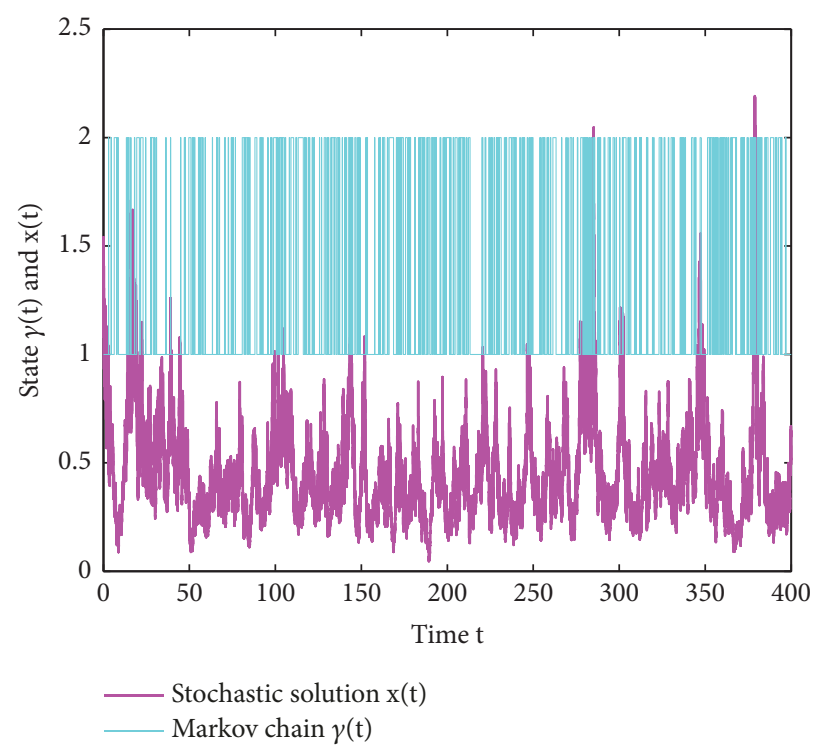

Figure 3: Stochastic trajectories of model (6) with parameters in Example 3.

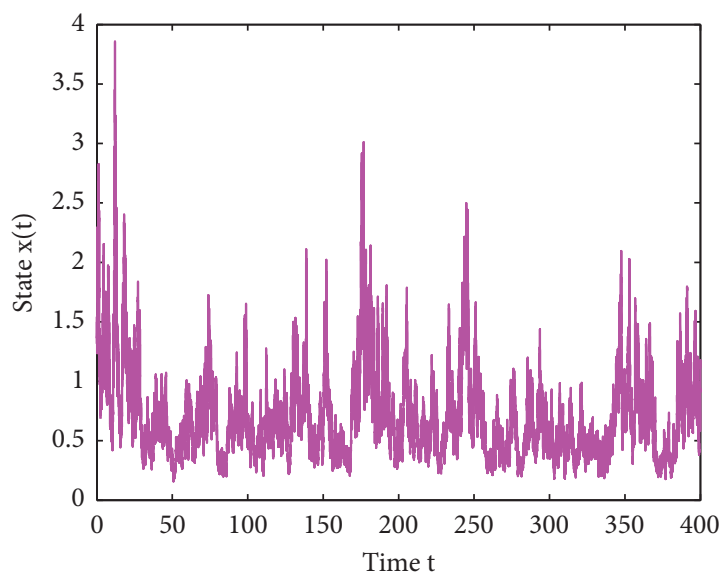

Stochastic solution $\mathrm{x}(\mathrm{t})$

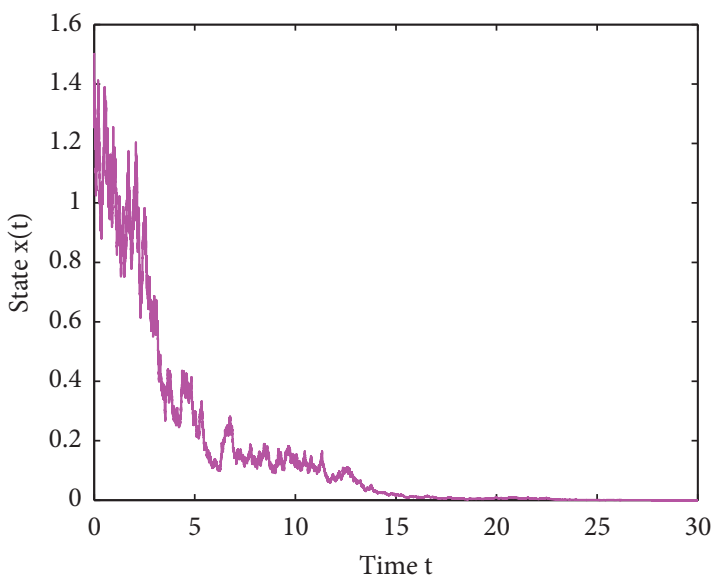

Stochastic solution $\mathrm{x}(\mathrm{t})$

FIGURE 4: Stochastic trajectories of subsystems (109) and (110).

$-0.125<0$. From Corollary 4.6 in [26], subsystem (110) is stochastically extinct. Figure 4 clearly supports these results.

In Figure 4, (i) left panel shows stochastic trajectory of subsystem (109); (ii) right panel shows stochastic trajectory of subsystem (110).

Example 3 shows that if the overall system consists of permanent subsystems and nonpermanent subsystems, the overall system can be permanent. Hence, switching component can add some interesting behavior to hybrid systems.

\section{Conclusions}

In this paper, we study the stochastic permanence and asymptotic estimations of solution to a general stochastic delay Gilpin-Ayala system with Markovian switching. Compared with the models in the literatures, (6) provides a more realistic system of the population dynamics. However, the delays and Markovian switching mechanism make the task more complicated to deal with. We overcome difficulties by constructing suitable Lyapunov functionals and using some analysis technique. Under some suitable conditions, (6) still retains some well properties, for example, the existence of global positive solution and persistence and asymptotic estimations. Further, the results show that switching component can add some interesting behavior to hybrid systems driven by Markov chain. In addition, some interesting topics merit further consideration. One may propose stability distribution, ergodicity, and extinction.

\section{Data Availability}

No data were used to support this study. 


\section{Conflicts of Interest}

The authors declare that they have no conflicts of interest.

\section{Authors' Contributions}

All the authors contributed equally and significantly in writing this paper. All authors read and approved the final manuscript.

\section{Acknowledgments}

This work was supported by the National Natural Science Foundation of China (no. 11471197), the Natural Science Foundation of Shanxi Province (no. 201801D21009), and the Natural Science Foundation of Lvliang University (no. ZRXN201610).

\section{References}

[1] M. E. Gilpin and F. J. Ayala, "Global models of growth and competition," Proceedings of the National Acadamy of Sciences of the United States of America, vol. 70, no. 12, pp. 3590-3593, 1973.

[2] M. E. Gilpin and F. J. Ayala, "Schoener's model and Drosophila competition," Theoretical Population Biology, vol. 9, no. 1, pp. 1214,1976

[3] X. R. Mao, Stochastic Differential Equations and Applications, Horwood publishing, Chichester, UK, 2007.

[4] A. Lahrouz and A. Settati, "A note on stochastics Gilpin-Ayala population model with dispersal," Differential Equations and Dynamical Systems, vol. 25, no. 3, pp. 417-430, 2017.

[5] M. Jovanović and M. Krstić, "Extinction in stochastic perdatorprey population model with Allee effect on prey," Discrete and Continuous Dynamical Systems, Series B, vol. 22, no. 7, pp. 26512667, 2017.

[6] T. Caraballo, M. El Fatini, R. Pettersson, and R. Taki, "A stochastic SIRI epidemic model with relapse and media coverage," Discrete and Continuous Dynamical Systems - Series B, vol. 23, no. 8, pp. 3483-3501, 2018.

[7] M. Liu and M. Fan, "Permanence of stochastic Lotka-Volterra systems," Journal of Nonlinear Science, vol. 27, pp. 425-452, 2017.

[8] M. Liu and K. Wang, "Stationary distribution, ergodicity and extinction of a stochastic generalized logistic system," Applied Mathematics Letters, vol. 25, no. 11, pp. 1980-1985, 2012.

[9] D. S. Li, "The stationary distribution and ergodicity of a stochastic generalized logistic system," Statistics Probability Letters, vol. 83, no. 2, pp. 580-583, 2013.

[10] X. W. Yu, S. L. Yuan, and T. H. Zhang, "Persistence and ergodicity of a stachastic single species model with Allee effect under regime swiching," Communications in Nonlinear Science and Numerical Simulation, vol. 59, pp. 359-374, 2018.

[11] M. Liu and K. Wang, "Asymptotic properties and simulations of a stochastic logistic model under regime switching," Mathematical and Computer Modelling, vol. 54, no. 9-10, pp. 2139-2154, 2011.

[12] M. Liu and K. Wang, "Asymptotic properties and simulations of a stochastic logistic model under regime switching II," Mathematical and Computer Modelling, vol. 55, no. 3-4, pp. 405418, 2012.
[13] A. Settati and A. Lahrouz, "On stochastic Gilpin-Ayala population model with Markovian switching," BioSystems, vol. 130, pp. 17-27, 2015.

[14] A. Settati and A. Lahrouz, "Stability and ergodicity of a stochastic Gilpin-Ayala model under regime switching on patches," International Journal of Biomathematics, vol. 10, no. 6, Article ID 1750090, pp. 617-625, 2017.

[15] M. Liu, "Dynamics of a stochastic regime-switching predatorprey model with modified Leslie-Gower Holling-type II schemes and prey harvesting," Nonlinear Dynamics, vol. 96, no. 1, pp. 417-442, 2019.

[16] M. Liu, J. Yu, and P. S. Mandal, "Dynamics of a stochastic delay competitive model with harvesting and Markovian switching," Applied Mathematics and Computation, vol. 337, pp. 335-349, 2018.

[17] M. Liu and M. Deng, "Permanence and extinction of a stochastic hybrid model for tumor growth," Applied Mathematics Letters, vol. 94, pp. 66-72, 2019.

[18] A. Settati, S. Hamdoune, A. Imlahi, and A. Akharif, "Extinction and persistence of a stochastic Gilpin-Ayala model under regime switching on patches," Applied Mathematics Letters, vol. 90, pp. 110-117, 2019.

[19] Y. Kuang, Delay Differential Equations with Applications in Population Dynamics, Academic Press, New York, NY, USA, 1993.

[20] A. Bahar and X. Mao, "Stochastic delay popuation dynamics," Pure and Appied Mathematics, vol. 11, no. 4, pp. 377-400, 2004.

[21] C. Shi, "Some new results on the permanence of a logistic type system with time delays," Annals of Differential Equations. Weifen Fangcheng Niankan, vol. 22, no. 4, pp. 560-568, 2006.

[22] M. Vasilova and M. Jovanović, "Stochastic Gilpin-Ayala competition model with infinite delay," Applied Mathematics and Computation, vol. 217, no. 10, pp. 4944-4959, 2011.

[23] K. Zhao and Y. Ren, "Existence of positive periodic solutions for a class of Gilpin-Ayala ecological models with discrete and distributed time delays," Advances in Difference Equations, vol. 331, pp. 1-13, 2017.

[24] J. Baštinec, H. Demchenko, J. Diblik, and D. Y. Khusainov, "Exponential stability of linear discrete systems with multiple delays," Discrete Dynamics in Nature and Society, vol. 2018, Article ID 9703919, 7 pages, 2018.

[25] Y. Y. Liu and T. Hou, "Exponential stability and Robust $\mathrm{H}_{\infty}$ control for discrete-time time-delay infinite Markov jump systems," Discrete Dynamics in Nature and Society, vol. 2018, Article ID 3676083, 9 pages, 2018.

[26] Z. Wu, H. Huang, and L. Wang, "Stochastic delay logistic model under regime switching," Abstract and Applied Analysis, vol. 2012, Article ID 241702, 26 pages, 2012.

[27] X. Mao and C. Yuan, Stochastic Differential Equations with Markovian Switching, Imperial College Press, London, UK, 2006. 


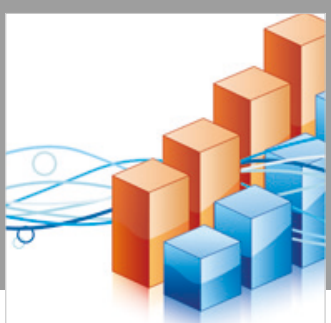

Advances in

Operations Research

\section{-n-m}
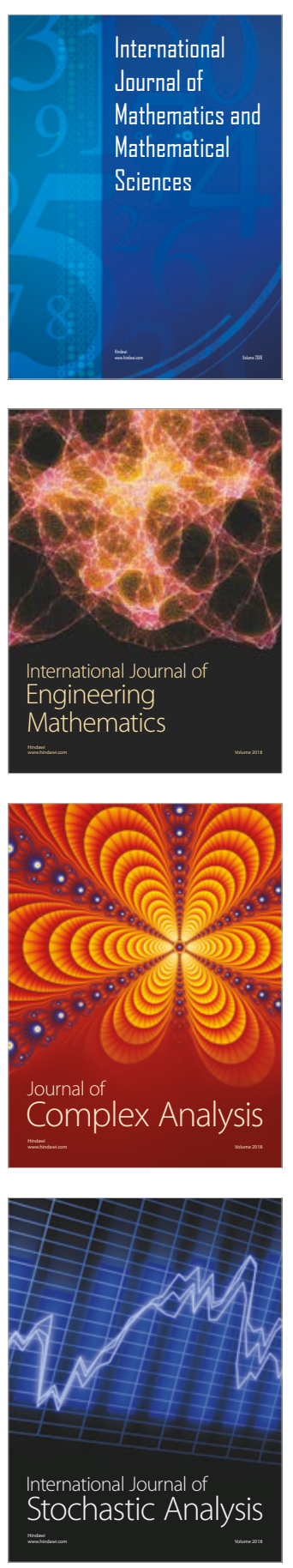
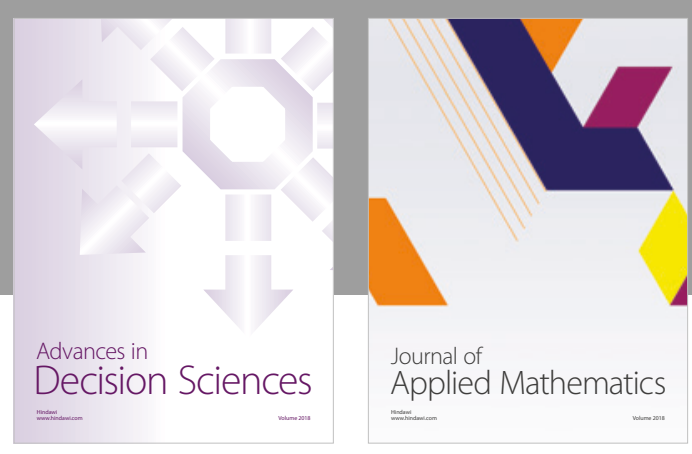

Journal of

Applied Mathematics
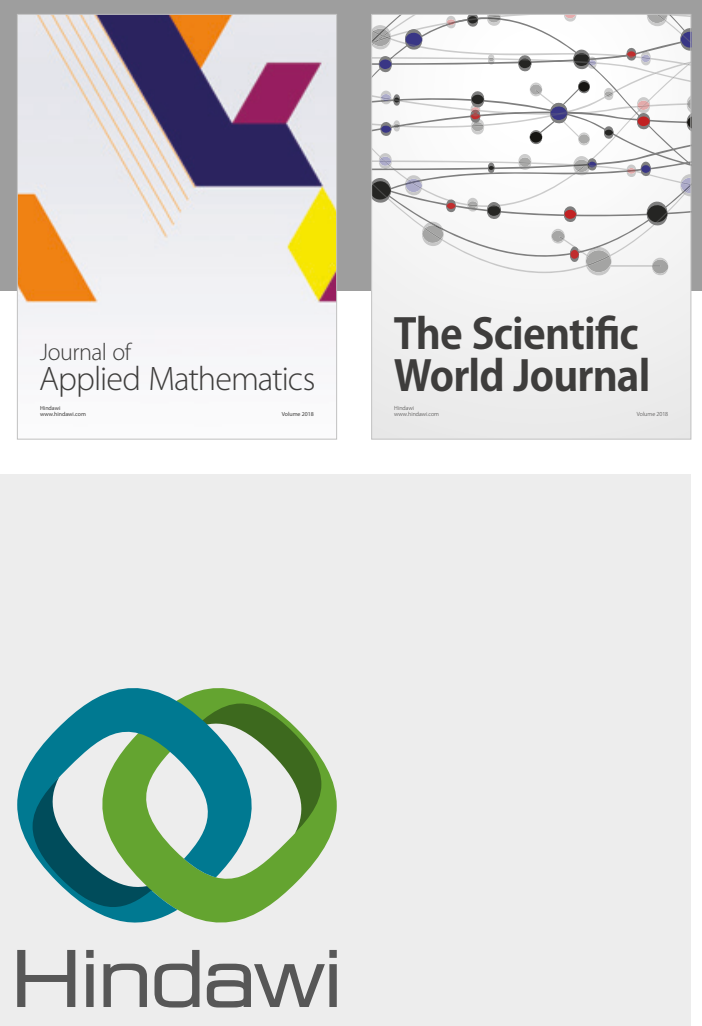

Submit your manuscripts at

www.hindawi.com

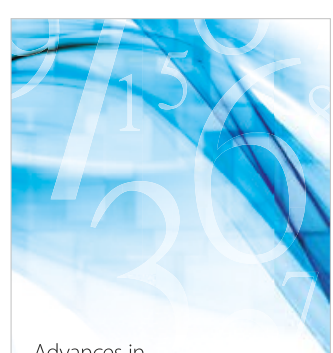

Advances in
Numerical Analysis
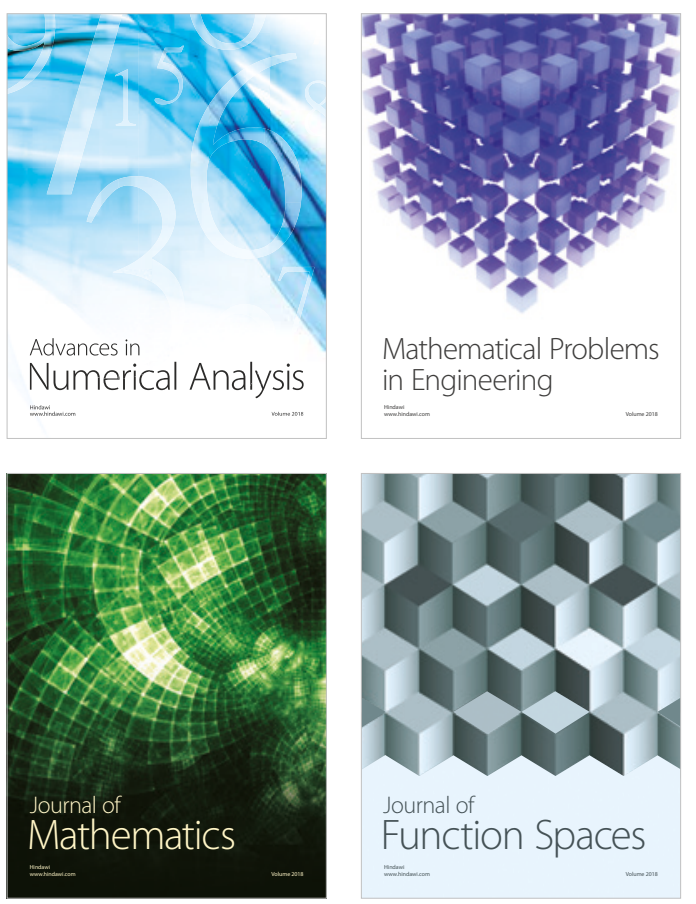

Mathematical Problems in Engineering

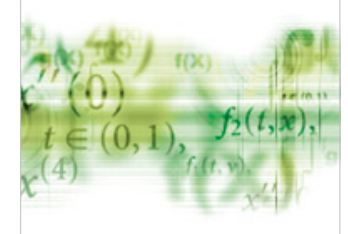

International Journal of

Differential Equations

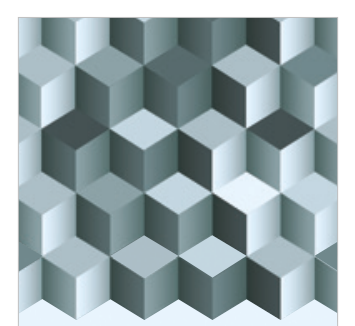

Journal of

Function Spaces

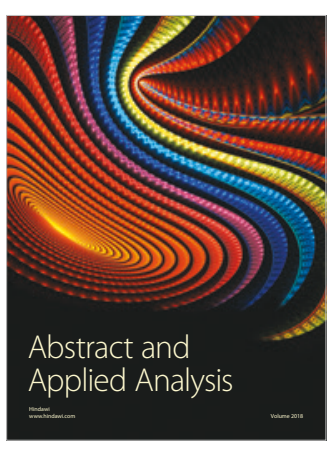

The Scientific

World Journal

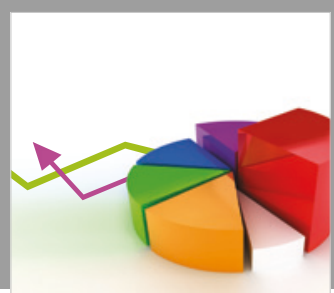

Journal of

Probability and Statistics
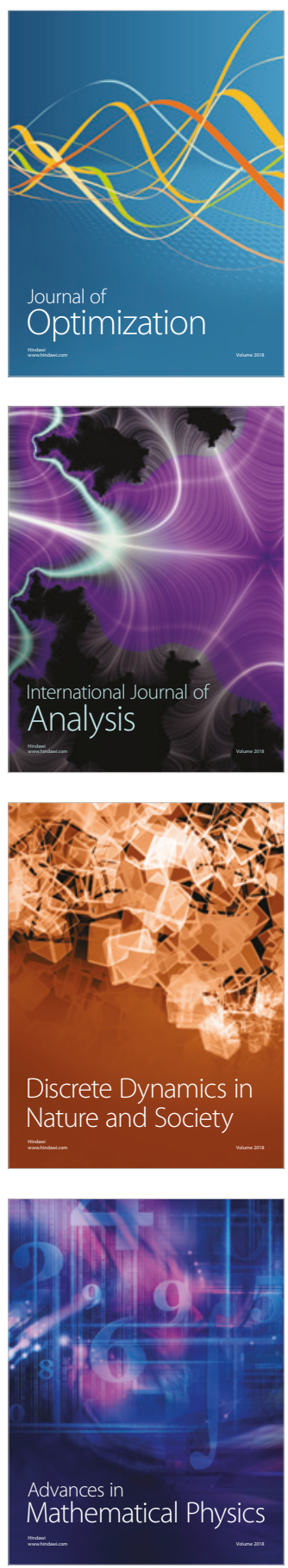